\title{
A spatially resolved study of photoelectric heating and [C II] cooling in the LMC
}

\section{Comparison with dust emission as seen by SAGE}

\author{
D. Rubin ${ }^{1}$, S. Hony ${ }^{1}$, S. C. Madden ${ }^{1}$, A. G. G. M Tielens ${ }^{2}$, M. Meixner ${ }^{3}$, R. Indebetouw ${ }^{4}$, W. Reach ${ }^{5}$, A. Ginsburg ${ }^{6}$, \\ S. Kim ${ }^{7}$, K. Mochizuki ${ }^{8}$, B. Babler ${ }^{9}$, M. Block ${ }^{10}$, S. B. Bracker ${ }^{9}$, C. W. Engelbracht ${ }^{10}$, B.-Q. For ${ }^{10}$, K. Gordon ${ }^{10}$, \\ J. L. Hora ${ }^{11}$, C. Leitherer ${ }^{3}$, M. Meade ${ }^{9}$, K. Misselt ${ }^{10}$, M. Sewilo ${ }^{3}$, U. Vijh ${ }^{3}$, and B. Whitney ${ }^{12}$
}

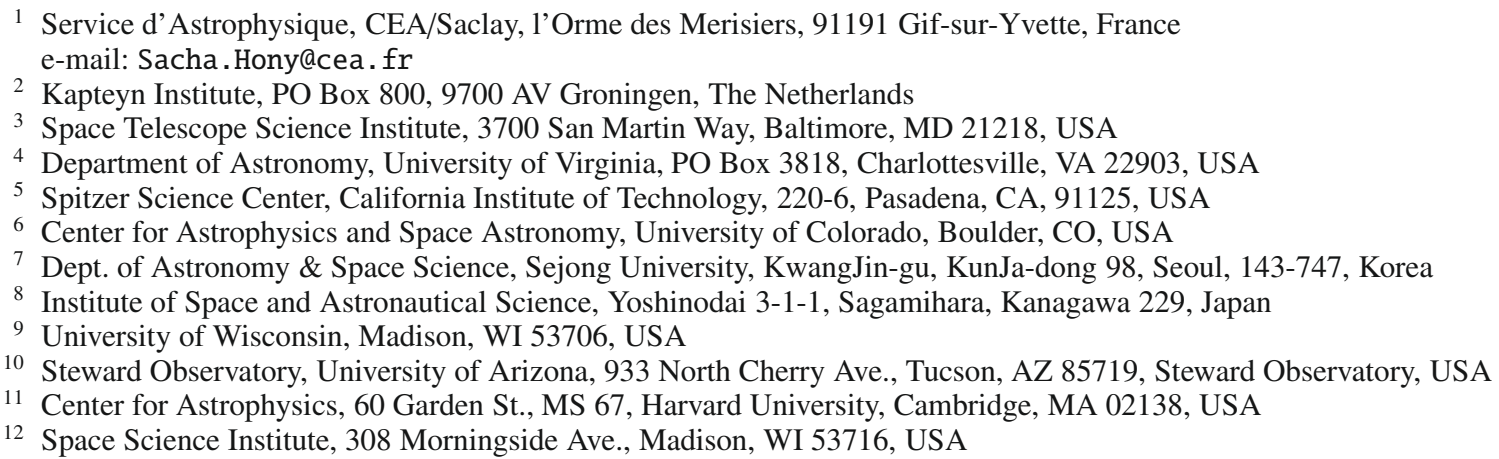

received 16 September 2008/ Accepted 25 November 2008

ABSTRACT

\begin{abstract}
Context. Photoelectric heating is a dominant heating mechanism for many phases of the interstellar medium. We study this mechanism throughout the Large Magellanic Cloud (LMC).

Aims. We aim to quantify the importance of the [C II] cooling line and the photoelectric heating process of various environments in the LMC and to investigate which parameters control the extent of photoelectric heating.

Methods. We use the BICE [C II] map and the Spitzer/SAGE infrared maps. We examine the spatial variations in the efficiency of photoelectric heating: photoelectric heating rate over power absorbed by grains, i.e. the observed [C II] line strength over the integrated infrared emission. We correlate the photoelectric heating efficiency and the emission from various dust constituents and study the variations as a function of $\mathrm{H} \alpha$ emission, dust temperatures, and the total infrared luminosity. The observed variations are interpreted in a theoretical framework. From this we estimate radiation field, gas temperature, and electron density.

Results. We find systematic variations in photoelectric efficiency. The highest efficiencies are found in the diffuse medium, while the lowest coincide with bright star-forming regions ( 1.4 times lower). The $[\mathrm{C}$ II] line emission constitutes $1.32 \%$ of the far infrared luminosity across the whole of the LMC. We find correlations between the [C II] emission and ratios of the mid infrared and far infrared bands, which comprise various dust constituents. The correlations are interpreted in light of the spatial variations of the dust abundance and by the local environmental conditions that affect the dust emission properties. As a function of the total infrared surface brightness, $S_{\mathrm{TIR}}$, the [C II] surface brightness can be described as: $S_{\text {[CII] }}=1.25 S_{\mathrm{TIR}}^{0.69}\left[10^{-3} \mathrm{erg} \mathrm{s}^{-1} \mathrm{~cm}^{-2} \mathrm{sr}^{-1}\right.$ ], for $S_{\mathrm{TIR}} \gtrsim 3.2 \times 10^{-4} \mathrm{erg} \mathrm{s}^{-1} \mathrm{~cm}^{-2} \mathrm{sr}^{-1}$. We provide a simple model of the photoelectric efficiency as a function of the total infrared luminosity. We find a power-law relation between radiation field and electron density, consistent with other studies. The [C II] emission is well-correlated with the $8 \mu \mathrm{m}$ emission, suggesting that the polycyclic aromatic hydrocarbons play a dominant role in the photoelectric heating process.
\end{abstract}

Key words. galaxies: Magellanic Clouds - ISM: dust, extinction - infrared: galaxies - ISM: lines and bands

\section{Introduction}

The structure and evolution of the interstellar medium (ISM) is largely dependent upon the thermal processes taking place (Goldsmith et al. 1969; de Jong 1977; McKee \& Ostriker 1977; Draine 1978; Ferriere et al. 1988). This, in turn, shapes the evolution of galaxies as a whole, as the constituents of the ISM are responsible for the characteristics of incipient stellar generations. Therefore, an understanding of the agents which dominate the heating and cooling of interstellar gas is of fundamental importance.

A dominant heating source of the ISM of galaxies is the photoelectric (PE) emission of interstellar dust grains. Absorption of a far-ultraviolet (FUV) photon by a dust grain may result in the ejection of an energetic electron which heats interstellar gas via collisions. Photoelectric emission as a heating mechanism of the ISM was first proposed by Spitzer (1948) and later revisited by Watson (1972) and de Jong (1977). Since then, it has been found that the process dominates the heating of a range of 
interstellar media: neutral atomic gas clouds, the photodissociation regions (PDRs), and the warm inter-cloud medium (e.g. Maciel \& Pottasch 1982; Weingartner \& Draine 2001)

The PE heating process has received much theoretical attention (e.g. Watson 1972; de Jong 1977; Draine 1978; Tielens \& Hollenbach 1985b; Bakes \& Tielens 1994; Dwek \& Smith 1996; Weingartner \& Draine 2001). Due to grain charging, PE heating efficiency is highly dependent on the physical conditions which determine the ionisation and recombination rates. Specifically, it depends on the FUV radiation field, gas temperature and electron density. In turn, the extent of grain charging and therefore the efficiency of PE heating is also highly dependent on the grain species involved.

The $\mathrm{C}^{+}$fine structure transition $\left({ }^{2} \mathrm{P}_{3 / 2}-{ }^{2} \mathrm{P}_{1 / 2}\right)$ is the dominant coolant of the diffuse ionised and diffuse atomic gas as well as in PDRs (Dalgarno \& McCray 1972; Tielens \& Hollenbach 1985b,a; Stacey et al. 1991; Madden et al. 1993; Petuchowski \& Bennett 1993; Heiles 1994). Several reasons account for its dominance: carbon is the fourth most abundant element and it has an ionisation potential of $11.3 \mathrm{eV}$, less than that of $\mathrm{H}$. This $\mathrm{C}^{+}$transition is also easy to excite as it has a relatively low excitation temperature $(\sim 92 \mathrm{~K})$. Thus, it is able to cool warm neutral gas $\left(T \cong 30-10^{4} \mathrm{~K}\right)$ whereas other species can not (Tielens \& Hollenbach 1985b,a; Wolfire et al. 1990). The efficiency of the $\mathrm{C}^{+}$as a coolant is dependent upon environment. When temperatures or densities are high, other lines, primarily [OI] $63 \mu \mathrm{m}$, participate in the cooling process (Tielens \& Hollenbach 1985b,a; Hollenbach et al. 1991). The critical density of the $\mathrm{C}^{+}$transition is relatively low $\left(\sim 3 \times 10^{3} \mathrm{~cm}^{-3}\right)$. At densities above the critical density or temperature above $92 \mathrm{~K}$ the cooling by the $[\mathrm{C} \mathrm{II}]$ line saturates and its importance as coolant diminishes.

An observational study of PE heating and gas cooling requires $[\mathrm{C} \mathrm{II}]^{1}$ and infrared (IR) observations, covering wavelengths tracing the variety of dust components in the ISM, and ideally of sufficient spatial resolution to separate environments, because of the dependence of the process on environment and composition. Because of its proximity, the Large Magellanic Cloud (LMC) is an obvious candidate for a study of these processes. We undertake such a study using the Spitzer legacy program: Surveying the Agents of a Galaxy's Evolution (SAGE Meixner et al. 2006; Bernard et al. 2008), and the Balloonborne Infrared Carbon Explorer mission (BICE Mochizuki et al. 1994). SAGE fully mapped the LMC at high spatial resolution from 3 to $160 \mu \mathrm{m}$ while the latter offers a [C II] map of the entire LMC. These datasets offer advantages over previous work because of their enhanced spatial resolution, wavelength coverage, and sensitivity. Prior studies using NASA's Kuiper Airborne Observatory (KAO) and the Long Wavelength Spectrometre (LWS) aboard the Infrared Space Observatory (ISO) examined [C II] emission integrated across whole galaxies (e.g. Stacey et al. 1991; Madden et al. 1993; Malhotra et al. 2001), or in specific regions within galaxies (e.g. Stutzki et al. 1988; Meixner et al. 1992; Poglitsch et al. 1995; Israel et al. 1996; Madden et al. 1997). There have not been many [C II] studies which probe the range of different phases of the ISM. Moreover, even though PE efficiency is believed to be a strong function of grain size, there have not been many prior observational studies of the effect of PE heating due to distinct grain populations. Most studies have considered the total PE heating across all grain populations

\footnotetext{
1 Throughout this paper, we utilise the following notation: we refer to the fine structure transition with $\mathrm{C}^{+}$and to the emission line that it produces with $[\mathrm{C} \mathrm{II}]$.
}

because they did not have access to bands which trace the emission from distinct grain populations.

The composition of the ISM of the LMC makes it an interesting laboratory because it has a low metallicity; $Z \cong 0.3-0.5 Z_{\odot}$ (Westerlund 1997) and $\mathrm{Z} \cong 0.25 \mathrm{Z}_{\odot}$ (Dufour 1984). The presence of metals, in the form of dust, is integral to the PE effect. How does the low metallicity lower dust abundance affect PE heating?

Moreover, it is known from observational studies that the LMC and other low metallicity galaxies have a dearth of polycyclic aromatic hydrocarbons (PAHs) compared to Galactic values (Sakon et al. 2006; Vermeij et al. 2002b; Madden et al. 2006; Wu et al. 2006; Galliano et al. 2007; Engelbracht et al. 2008). This condition raises another question: since it is found theoretically that PAHs should play the greatest role in the PE heating process, how is the PE heating affected in a galaxy with a prominent dearth of PAHs?

In contrast to the metallicity argument given above, it is found from observations that the $\mathrm{C}^{+}$coolant plays an even more important role at low metallicity than it does at high metallicity, contributing up to $\sim 10$ times more to the far-infrared (FIR) emission (Poglitsch et al. 1995; Israel et al. 1996; Madden et al. 1997). The larger relative strength has been explained by the clumpy nature of the ISM in low metallicity galaxies. Observations of $[\mathrm{C} \mathrm{II}] / \mathrm{CO}$ show that for low metallicity galaxies, this ratio can be 10-30 times higher than for normal galaxies or active galaxies (e.g. Madden 2000). The far UV radiation penetrates deeper into the molecular cloud at low $Z$, for the same $A_{\mathrm{V}}$, leaving a smaller $\mathrm{CO}$ core and a larger $\mathrm{C}^{+}$emitting envelope. The result is a preponderance of clumps with $\mathrm{CO}$ cores of larger exposed surface area. As the $\mathrm{C}^{+}$resides primarily in the envelopes of the clouds the increase in surface area results in a higher ratio of [C II]/CO emission (e.g. Röllig et al. 2007).

The aim of this paper is to explore the qualitative behaviour and observationally quantify the extent of PE heating and [C II] cooling in relation to environment. The format of this paper is as follows: Sect. 2 reviews the observational details of the data used in this study. Section 3 presents the data treatment process and the final images.

Section 4 quantifies the importance of the [C II] coolant globally across the LMC. In Sect. 5 we examine PE heating as a function of environment based on the $\mathrm{H} \alpha$ surface brightness and Sect. 6 explores the variations in [C II] emission within the LMC using the $\mathrm{H} \alpha$ criterion and dust temperature. To explore the correlation between $[\mathrm{C} \mathrm{II}]$ emission and the emission from the various grain components in the LMC, Sect. 7 compares the spatial distribution of the [C II] emission and the emission at various Spitzer bands. The key-concepts of PE heating are reviewed in Sect. 7.2, which are applied in Sect. 8 to describe the PE heating as a function of radiation field within the LMC. Using the observed relation we calculate electron densities, and find a correlation between radiation field and electron density (Sect. 9). The paper concludes with Sect. 10, an analysis of the dependence of PE heating on grain population. We study the qualitative behaviour of the extent of PE heating on grain population, and we also quantify the contributions to PE heating from the various grain populations.

\section{Observations}

Relevant observational details of all the maps used in this study (the [C II], Spitzer and $\mathrm{H} \alpha$ maps) are summarised in Table 1. 
Table 1. Summary of data used in this study.

\begin{tabular}{lcccccc}
\hline \hline Line/Band & {$[\mathrm{CII}]$} & $8 \mu \mathrm{m}$ & $24 \mu \mathrm{m}$ & $70 \mu \mathrm{m}$ & $160 \mu \mathrm{m}$ & $\mathrm{H} \alpha$ \\
\hline Mission & BICE & SAGE & SAGE & SAGE & SAGE & SHASSA \\
$1 \sigma$ limit [MJy sr-1 & - & 0.2 & 0.2 & 1.0 & 2.0 & - \\
$1 \sigma$ limit $\left[10^{-5} \mathrm{erg} \mathrm{s}^{-1} \mathrm{~cm}^{-2} \mathrm{sr}^{-1}\right]^{a}$ & 0.47 & 3.0 & 0.7 & 1.0 & 0.8 & $1.21 \times 10^{-2}$ \\
$1 \sigma$ limit $\left[\mathrm{MJy} \mathrm{sr}^{-1}{ }^{a}\right.$ & - & $4 \times 10^{-4}$ & $4 \times 10^{-4}$ & $4 \times 10^{-3}$ & $2 \times 10^{-2}$ & - \\
$1 \sigma$ limit $\left[10^{-5} \mathrm{erg} \mathrm{s}^{-1} \mathrm{~cm}^{-2} \mathrm{sr}^{-1}\right]^{a}$ & 0.47 & $6 \times 10^{-3}$ & $1 \times 10^{-3}$ & $5 \times 10^{-3}$ & $1 \times 10^{-2}$ & $4.45 \times 10^{-5}$ \\
Survey Area & $6^{\circ} \times 10^{\circ}$ & $7.1^{\circ} \times 7.1^{\circ}$ & $7.8^{\circ} \times 7.8^{\circ}$ & $7.8^{\circ} \times 7.8^{\circ}$ & $7.8^{\circ} \times 7.8^{\circ}$ & $13^{\circ} \times 13^{\circ}$ \\
Beamsize ["] & 894 & 2 & 6 & 18 & 40 & 240 \\
Linear size [pc] & $\sim 225$ & $\sim 0.5$ & $\sim 1.5$ & $\sim 4.5$ & $\sim 10$ & $\sim 60$ \\
Reference & {$[1]$} & {$[2]$} & {$[2]$} & {$[2]$} & {$[2]$} & {$[3]$} \\
\hline
\end{tabular}

${ }^{a}$ Sigma values after full data treatment, as outlined in Sect. 3. References: [1] Mochizuki et al. (1994); [2] Meixner et al. (2006); [3] Gaustad et al. (2001).

\subsection{The $[C I I]$ line}

The entire LMC was mapped in the $158 \mu \mathrm{m}[\mathrm{C}$ II] line by Mochizuki et al. (1994) during the BICE mission. The velocity integrated [C II] line surface brightness map was continuum subtracted by using a linear baseline and foreground subtracted using COBE data to estimate the Milky Way contribution. The measurements are calibrated against observations of M 17 carried out by Matsuhara et al. (1989). We compare several regions of the 30 Doradus complex mapped by Poglitsch et al. (1995) and Israel et al. (1996) aboard the KAO with the BICE map and find agreement better than $\sim 20 \%$. This is within the $30 \%$ calibration uncertainty of the BICE map as quoted by Mochizuki et al. (1994). The one $\sigma$ level for the BICE map as determined by Mochizuki et al. (1994) is $0.47 \times 10^{-5} \mathrm{erg} \mathrm{s}^{-1} \mathrm{~cm}^{-2} \mathrm{sr}^{-1}$.

The BICE map offers a $6^{\circ} \times 10^{\circ}$ field of view of the LMC (see also Figs. 1 and 2). The beam has a FWHM of $14.9^{\prime}$, corresponding to a linear size of $\sim 225 \mathrm{pc}$ at the distance of the LMC (50 kpc Feast 1999; Keller \& Wood 2006). The positional uncertainty is $\sim 6^{\prime}$ (Mochizuki et al. 1994). We have regrided the original map into pixels of $3^{\prime}(\sim 45 \mathrm{pc})$ in length.

A significant fraction of the pixels are noise dominated: $46.1 \%, 21.7 \%$ and $10.8 \%$ of all pixels in the final map are above are above 1,2 and $3 \sigma_{[\mathrm{C} I \mathrm{II}}$, respectively. The contours in Figs. 1 and 2 are spaced at multiples of $\sigma_{[\mathrm{C} \text { II] }}$. To sample the [C II] emission from the diffuse ISM, in our analysis throughout the rest of this paper, we consider pixels above the $2 \sigma_{[\mathrm{CI}}$ level.

\subsection{Infrared dust emission}

To probe dust properties and abundances, we use the SAGE infrared and mid-infrared survey of the LMC (Meixner et al. 2006). The survey offers a $7^{\circ} \times 7^{\circ}$ field of view of the LMC at effective wavelengths of 3.6, 4.5, 5.8, 8.0 (IRAC Fazio et al. 2004), 24.0, 70.0 and $160.0 \mu \mathrm{m}$ (MIPS Rieke et al. 2004). We use the 8,24, 70 and $160 \mu \mathrm{m}$ bands as the most important dust emission components can be traced primarily by these bands.

Zodiacal and Milky Way contamination light at MIPS wavelengths has been removed by doing off-source subtraction. The IRAC data have not been background subtracted as the background contribution is negligible compared to emission from the LMC. Averaging several regions located off the LMC in the $8 \mu \mathrm{m}$ image yields a background contribution of about $0.04 \mathrm{MJysr}^{-1}$. Such a level is indeed small compared to the mean value of about $0.46 \mathrm{MJysr}^{-1}$, for the $8 \mu \mathrm{m}$ image.

\section{3. $H_{\alpha}$ emission}

As a general way to distinguish physical environments within the LMC, we use an $\mathrm{H} \alpha$ surface brightness criterion. The $\mathrm{H} \alpha$ map used is part of the Southern H-alpha Sky Survey Atlas (Gaustad et al. 2001). The LMC is imaged in a single $13^{\circ} \times 13^{\circ}$ field and does not require mosaicing. The images are continuum subtracted and smoothed to a resolution of $4^{\prime}$. The sensitivity level of the $\mathrm{H} \alpha$ maps is about 0.5 Rayleigh $(=1.21 \times$ $10^{-7} \mathrm{erg} \mathrm{s}^{-1} \mathrm{~cm}^{2} \mathrm{sr}^{-1}$ at $\mathrm{H} \alpha$ ).

\section{Data treatment and presentation}

All IRAC, MIPS and $\mathrm{H} \alpha$ data were convolved to the lowest resolution data, the $[\mathrm{C} \mathrm{II}]$ map. The shape of [C II] beam is not precisely determined, and we therefore convolved the data with a Gaussian kernel with a FWHM of $14.9^{\prime}$, the FWHM of the BICE beam (Mochizuki et al. 1994). After convolution, the data were interpolated to match the $[\mathrm{C}$ II] pixel scheme.

To determine $\sigma$ values for the maps after the data treatment, we carried out a facsimile of the data treatment process with simulated Gaussian noise images. Standard deviations of the noise images after the full data treatment were measured, and are included in Table 1. We use those $\sigma$ values in our analysis.

Maps of the four Spitzer bands (convolved and re-gridded) with overlays of the [C II] contours are presented in Figs. 1 and 2. It is evident from Figs. $1 \mathrm{a}$ and $2 \mathrm{~b}$ that the $8 \mu \mathrm{m}$ and $160 \mu \mathrm{m}$ emission extends significantly into the bulk of the galaxy. Figures $1 \mathrm{~b}$ and 1a show that the $24 \mu \mathrm{m}$ and $70 \mu \mathrm{m}$ emission is much more concentrated toward H II regions such as 30 Dor and N11. More attention to the spatial distributions of the grain emission will be given in Sect. 7.

Note the slight offsets of the [C II] emission peaks from the IRAC and MIPS emission peaks (especially near N11), which can be larger than the $6^{\prime}$ pointing accuracy. Similar deviations were noted by Kim \& Reach (2002) in their analysis of atomic gas in conjunction with the BICE map. We have carefully checked for problems in the coordinate encoding used in the various maps. The observed displacements do not appear to be due to such problems and thus we conclude that there are real offsets between the peaks of emission of [C II] and IR emission, which can be as much as $100 \mathrm{pc}$. Indeed, the region near N11 is known to have most of its molecular material in the direction of the displacement as compared to the illuminating sources. Perhaps the observed displacement is due to offsets in the peak emission at the molecular interface, as such displacements are known to also occur in the Galaxy (e.g. Cesarsky et al. 1996). 

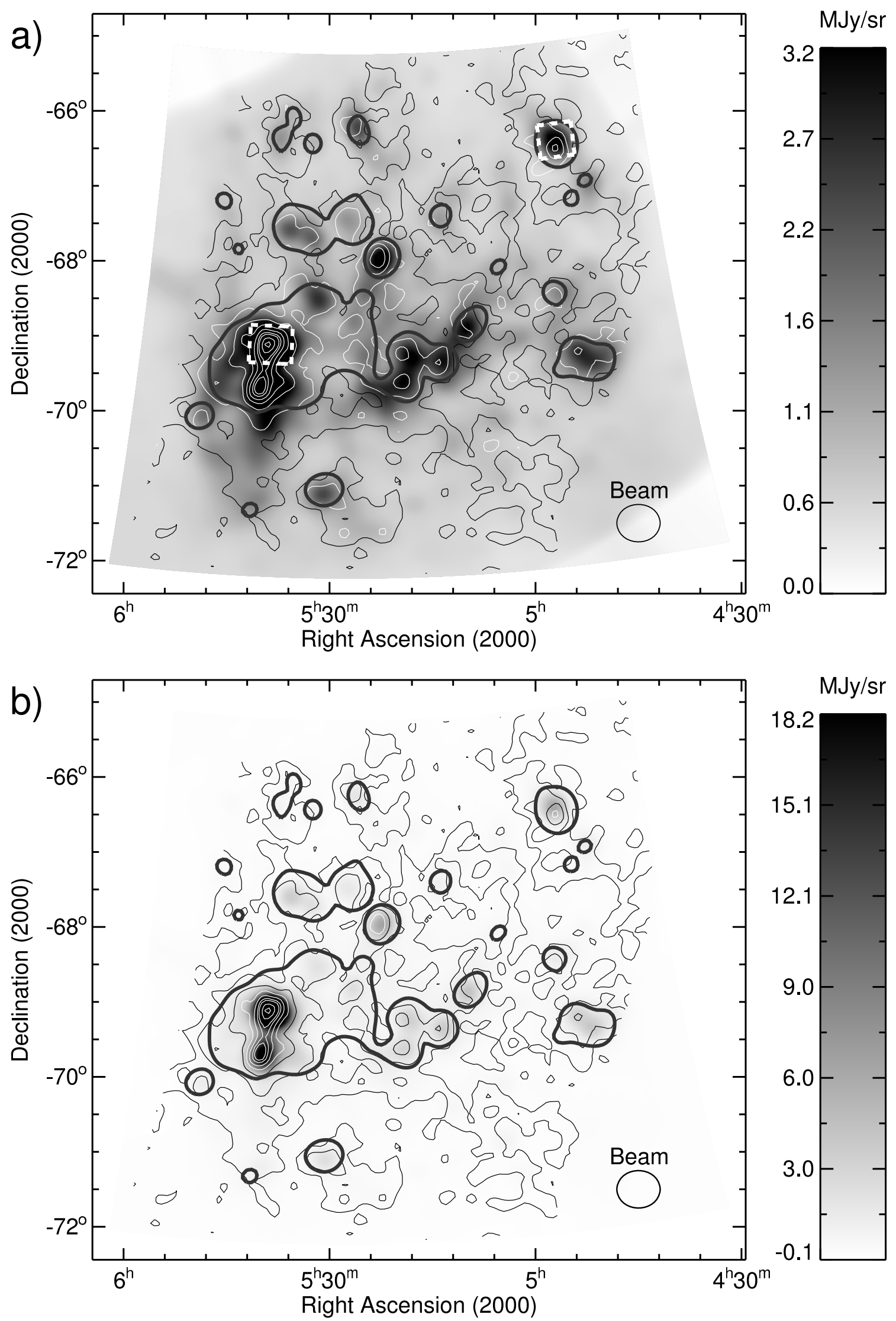

$\mathrm{MJy} / \mathrm{sr}$

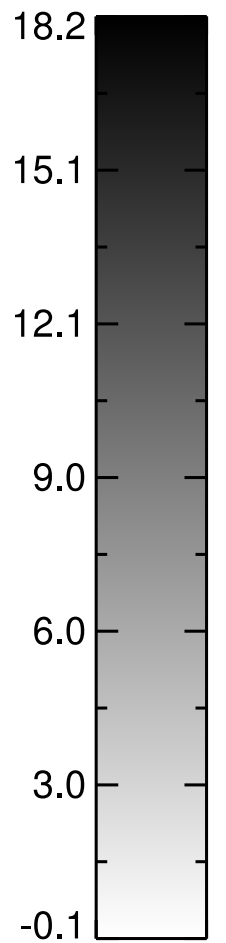

Fig. 1. $8 \mu \mathrm{m}$ a) and $24 \mu \mathrm{m} \mathrm{b}$ ) images convolved and re-gridded to the resolution and pixel scheme of the [C II] map. To enhance the contrast of the low level emission, each image has been truncated at 0.25 times the max of that particular image. The [C II] map is contoured over the images (thin grey and white contours) at levels of $1 \sigma_{[\mathrm{C} \text { II] }}$. The highest contours are white so that they can be distinguished from the underlying images. The thick grey lines enclose the regions which are defined as star forming given an $\mathrm{H} \alpha$ surface brightness criterion (Sect. 5). The dashed white boxes in a) indicate the regions used in the calculations for 30 Dor (southeast) and N11 (northwest) in Sects. 6 and 10. 


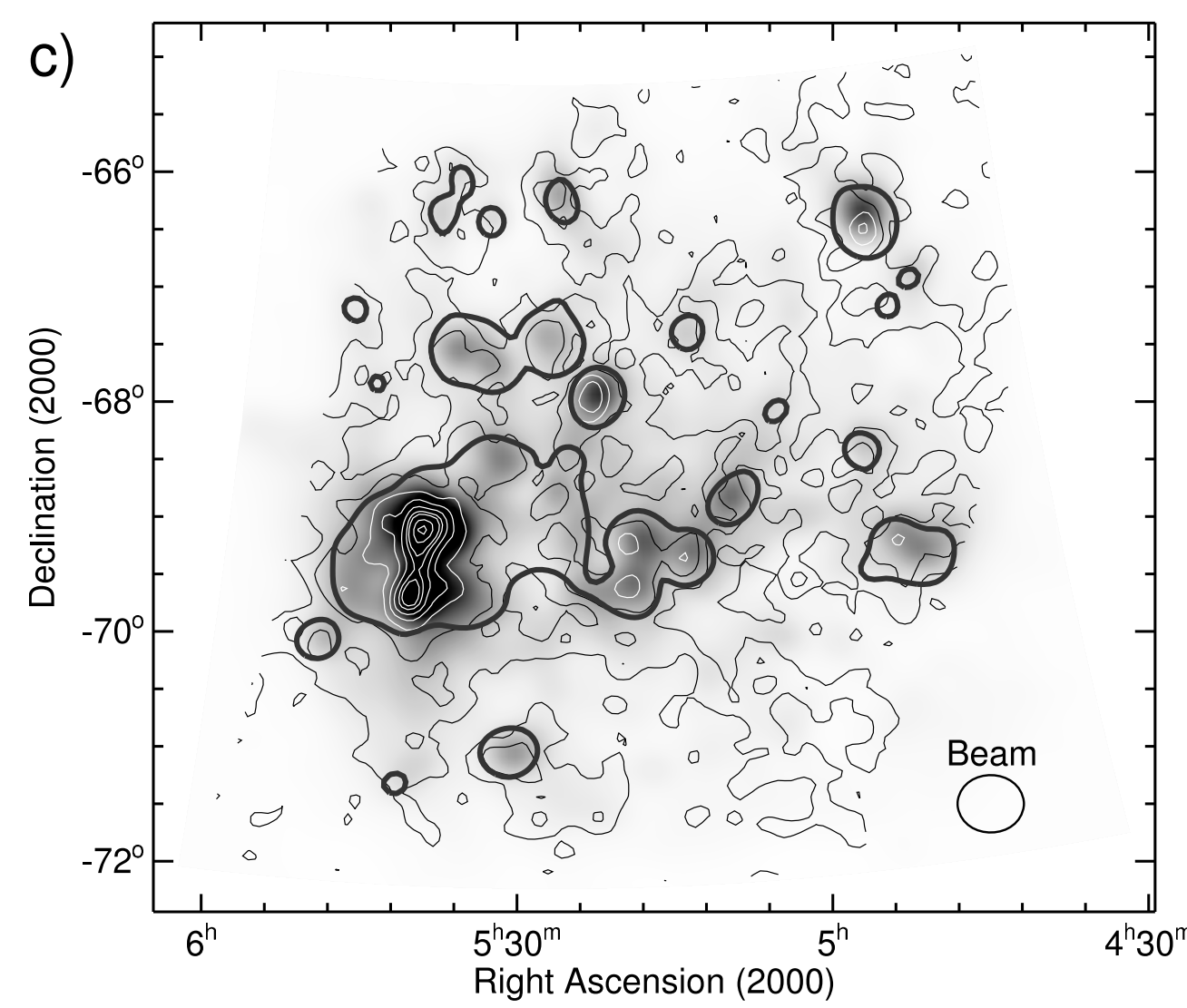

\section{$\mathrm{MJy} / \mathrm{sr}$}
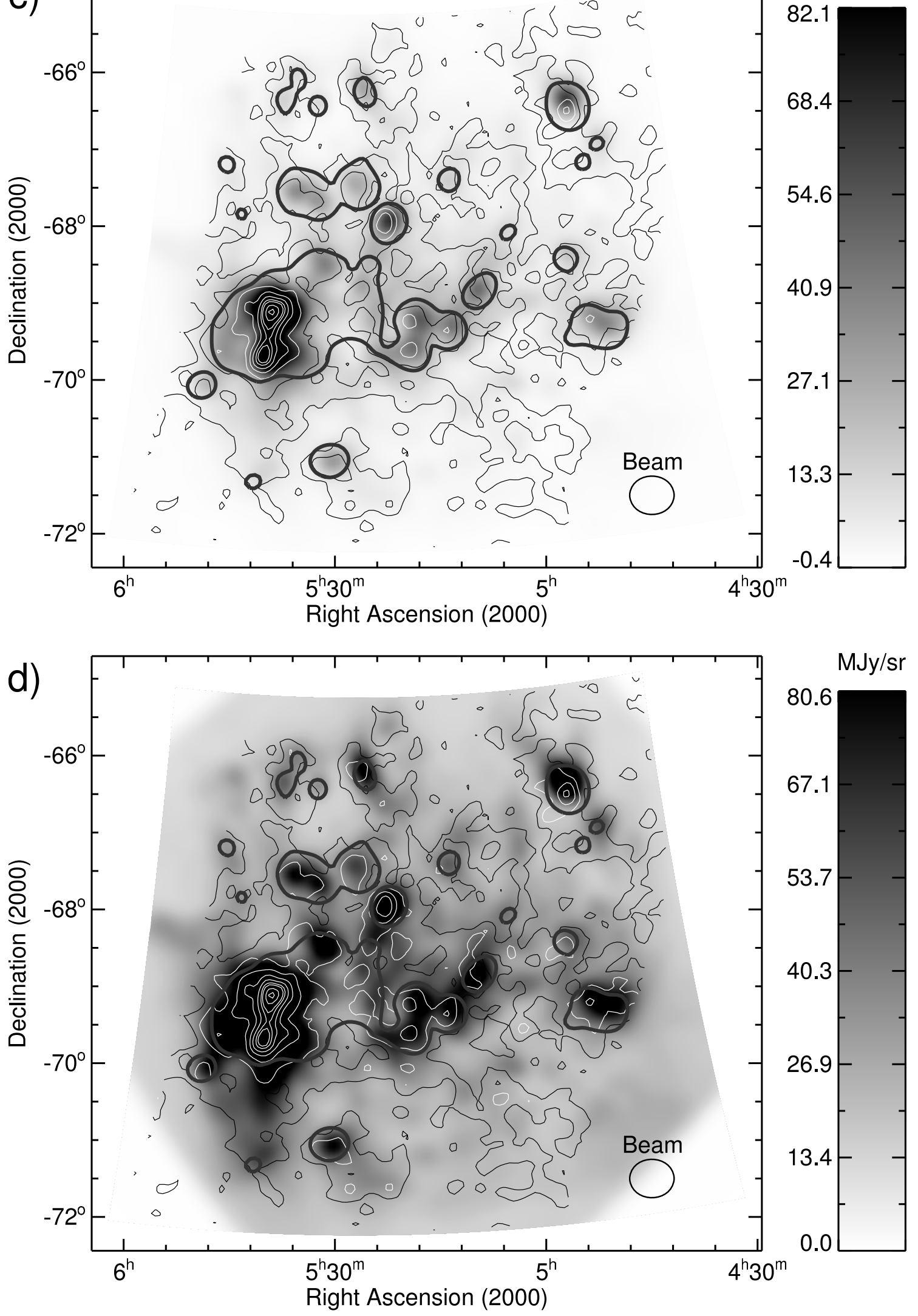

$\mathrm{MJy} / \mathrm{sr}$

80.6

67.1

(1)

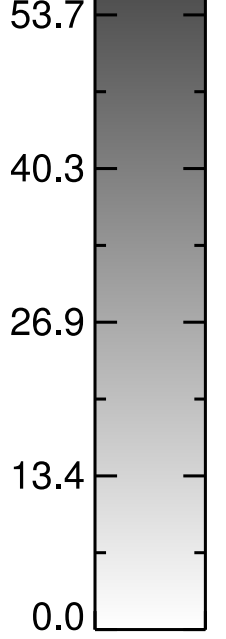

Fig. 2. $70 \mu \mathrm{m}$ a) and $160 \mu \mathrm{m} \mathrm{b}$ ) images convolved and re-gridded to the resolution and pixel scheme of the [C II] map. To enhance the contrast of the low level emission, the images have been truncated at 0.25 times the max of that particular value. The [C II] map is contoured over the images (thin grey and white contours) at levels of $1 \sigma_{[\mathrm{C} \text { II] }}$. The highest contours are white so that they can be distinguished from the underlying images. The thick grey lines enclose the regions which are defined as star forming given an $\mathrm{H} \alpha$ surface brightness criterion (Sect. 5). 


\section{4. [C II] emission globally across the LMC}

For comparison with other galaxies, we calculate the ratio of [C II] to the total infrared (TIR) integrated over the LMC. We adopt the expression of Dale \& Helou (2002) to calculate the TIR from the Spitzer the 24, 70 and $160 \mu \mathrm{m}$ filters, approximating the integrated 3 to $1000 \mu \mathrm{m}$ infrared surface brightness, $S_{\mathrm{TIR}}$ :

$S_{\mathrm{TIR}}=1.559 v S_{v, 24}+0.7686 v S_{v, 70}+1.347 v S_{v, 160}$.

The coefficients were derived from measured infrared SED shapes of a sample of galaxies observed by Spitzer. Draine \& Li (2007) also provide an equation for the TIR luminosity using a modified prescription of the Dale \& Helou (2002) SED model and incorporating the $8 \mu \mathrm{m}$ IRAC band as well as the MIPS bands. The difference between the two methods is less than $15 \%$. Since both prescriptions were made using SED properties from entire galaxies, it is important to check that they yield good estimates on smaller scales as well. We therefore compare the results from Eq. (1) with a straight integration over all the IRAC and MIPS bands. We find no systematic differences in the value of TIR as derived from the two methods for distinct environments, i.e. SF and the diffuse medium. Moreover, the values agree to within $\sim 10 \%$, which can most likely be accounted for by the differences in the precise wavelength ranges that both calculations consider.

The Spitzer definition of TIR emerged from the definition of $L_{\mathrm{FIR}}$ which was motivated by the IRAS bands (Helou 1986) and covers the FIR wavelength range from 42 to $122 \mu \mathrm{m}$. The difference between TIR and FIR has been observationally quantified by Hunter et al. (2001) who find that for irregular galaxies (such as the LMC), $L_{\mathrm{TIR}} / L_{\mathrm{FIR}} \approx 2$, (with a dispersion of only a few percent).

Integrated across the entire galaxy, we find that the total [C II] luminosity in the LMC is $(5.9 \pm 1.8) \times 10^{6} L_{\odot}$ assuming the distance to the LMC to be $50 \mathrm{kpc}$ (Feast 1999; Keller \& Wood 2006) $)^{2}$, consistent with Mochizuki et al. (1994) and Kim \& Reach (2002) who estimate $L_{[\mathrm{CII}}$ in the LMC to be $5.7 \times 10^{6} L_{\odot}$ and $6.5 \times 10^{6} L_{\odot}$ respectively. We find that the value of $L_{[\mathrm{C} \text { II] }} / L_{\mathrm{TIR}}$ is $0.46 \pm 0.14 \%$. Assuming a factor of 2 between TIR and FIR, $L_{[\mathrm{C} \text { II }]} / L_{\mathrm{FIR}}=0.9 \%$.

The relative contribution of [C II] to the integrated FIR has often been used to evaluate the global star formation activity in galaxies (e.g. Stacey et al. 1991). This value for the LMC is high compared to normal and gas rich galaxies which normally have values of $L_{[\mathrm{C} \text { II] }} / L_{\mathrm{FIR}}$ less than $1 \%$. Values of $L_{[\mathrm{C} \text { II] }} / L_{\mathrm{FIR}}=.1 \%$ to $0.5 \%$ are typical (Stacey et al. 1991; Malhotra et al. 1997, 2001 ); for the Milky Way $L_{[\mathrm{C} \text { II] }} / L_{\mathrm{FIR}} \sim 0.3 \%$ (Wright et al. $1991)$. Low metallicity galaxies can typically have $L_{[\mathrm{CII}]} / L_{\mathrm{FIR}}$ as high as $1 \%$ to $3 \%$ (Poglitsch et al. 1995; Israel et al. 1996; Madden et al. 1997; Madden 2000). This higher ratio is a consequence of the low metallicity: due to the reduced dust abundance, the overall mean free path of UV photons can be larger, resulting in a decrease in the FIR intensity arriving at the surfaces of the molecular clouds. To add to this effect, the lower dust attenuation results in the $\mathrm{C}^{+}$-emitting regions being larger as the photo-dissociating photons traverse a larger volume of the molecular cloud.

\footnotetext{
2 The contribution of the [C $\mathrm{II}]$ emission to the infrared emission is usually quoted in ratios of luminosities and we therefore adhere to this convention. We note, though, that we actually calculate values for this ratio with surface brightness. This is, however, equivalent to a ratio of luminosities assuming that both the $[\mathrm{CII}]$ and TIR are radiated isotropically.
}

\section{Distinction of physical environments}

We aim to study the PE heating and the [C II] cooling line as a function of environment. While the spatial resolution of $225 \mathrm{pc}$ results in some mixing of phases, we can still delineate distinct average conditions. We define two environments using the $\mathrm{H} \alpha$ surface-brightness. $\mathrm{H} \alpha$ emission is efficient at distinguishing between dense $\mathrm{H}$ II regions and diffuse media. This is because $\mathrm{H}$ II regions are $\mathrm{H} \alpha$ bright due to their high free-electron densities, which results in a high recombination rate. The $\mathrm{H} \alpha$ line is a tracer of the physical parameters which determine the degree of ionisation. That is, radiation field, temperature and density; all of which play critical roles in PE heating and the $\mathrm{C}^{+}$transition.

Kim \& Reach (2002) studied the LMC using the BICE [C II] map and made a similar distinction to separate the phases of the ISM. They studied the $\mathrm{C}^{+}$cooling rate for regions with an $\mathrm{H} \alpha$ surface brightness, $S_{\mathrm{H} \alpha}$, above and below $4.25 \times 10^{-5} \mathrm{erg} \mathrm{s}^{-1} \mathrm{~cm}^{-2} \mathrm{sr}^{-1}$. This number was based on the work of Kennicutt \& Hodge (1986). We considered using this criterion, but this causes several well known H II regions such as N41, N144 and N132 to be classified as diffuse. We conclude that the criterion should be lowered to better represent the different phases of the ISM. For a reformulation of the criterion, we examined the data of Kennicutt \& Hodge (1986). They photometrically observed $\mathrm{H}$ II regions in the LMC and tabulated the $\mathrm{H} \alpha$ surface brightness. A histogram of the distribution of these surface brightnesses, shows the rapid fall off at the lowest values, suggesting that the faint side of the distribution is noisedominated, while the bright side is comprised of reliable values. Indeed, Kennicutt \& Hodge (1986) warn that their measurements of H II regions with the lowest surface brightnesses are unreliable. We therefore take the value of the peak of the distribution, $1 \times 10^{-5} \mathrm{erg} \mathrm{s}^{-1} \mathrm{~cm}^{-2} \mathrm{sr}^{-1}$, as the lowest reliable surface brightness for an $\mathrm{H}$ II region, which we use to distinguish between physical environments in the LMC. Every pixel with an $\mathrm{H} \alpha$ surface brightness below this value we call "diffuse", and every pixel with an $\mathrm{H} \alpha$ surface brightness above it, we call "star forming" (SF).

The thick grey lines in Figs. 1 and 2 enclose the SF pixels, while the diffuse pixels reside outside the grey lines. One can see from this figure, that the SF regions correspond to the brightest H II regions, such as 30 Dor, N11 and the prominent $\mathrm{H}$ II regions along the LMC bar, while not extending too far into the diffuse medium. While we can not avoid including diffuse emission within these SF regions, most SF pixels are dominated by $\mathrm{H}$ II regions and PDRs. Likewise the regions we label diffuse will undoubtedly contain some denser ionised material, but will be dominated by the diffuse conditions.

To validate the threshold, we estimate the electron density $\left(n_{\mathrm{e}}\right)$ for the sets of SF and diffuse pixels under the Case B approximation. The approximation provides $S_{\mathrm{H} \alpha}$ which depends on electron density, size of the emitting regions and gas temperature (e.g. Valls-Gabaud 1998). For the estimation, we use the mean values of $S_{\mathrm{H} \alpha}$ for the $\mathrm{SF}$ and diffuse pixels, and a temperature of $10^{4} \mathrm{~K}$. The sizes of the emitting regions were determined by examining the original $\mathrm{H} \alpha$ images and visually determining the physical sizes of typical SF regions and the voids between them. For the SF pixels, we assume that the light is dominated by the emission from $\mathrm{H}$ II regions. The lengths determined were about $5^{\prime}$ and $15^{\prime}$ for the SF and diffuse regions respectively. For the SF pixels, we assume that the light is dominated by the emission from HII regions. This yields an average $n_{\mathrm{e}}$ of $\sim 100$ and $\sim 1 \mathrm{~cm}^{-3}$ for the SF and diffuse regions, respectively. The latter density is reasonable for densities of the 


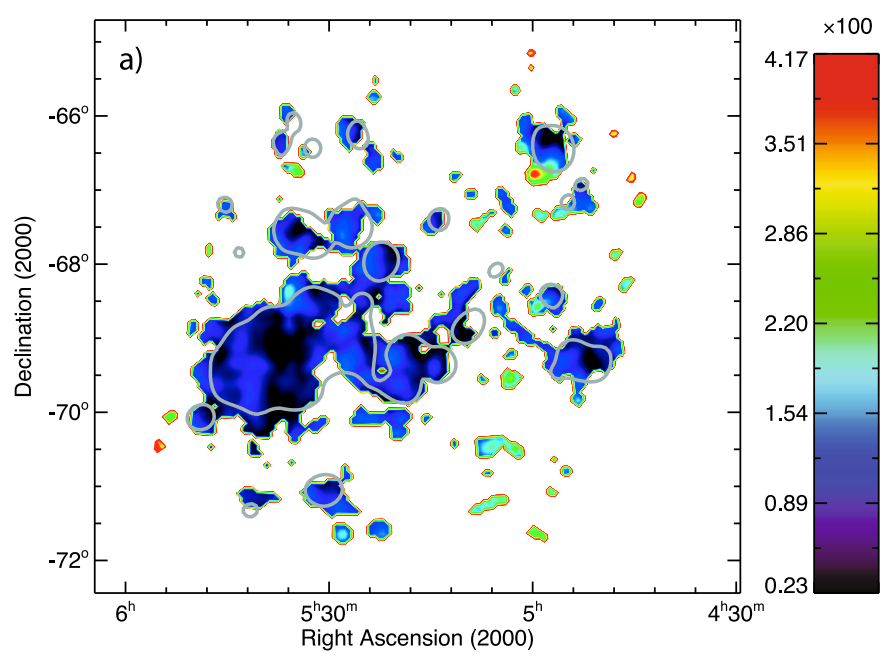

Fig. 3. Ratio map of $L_{\left[\mathrm{C}_{\text {II }}\right]} / L_{\mathrm{TIR}}($ left $)$. Pixels below $2 \sigma_{[\mathrm{C} \text { II] }}$ are set to white. The thick grey lines enclose the SF regions. (See online version for colour.)

warm ionised medium (Nordgren et al. 1992). The former is consistent with Peck et al. (1997), who find a mean electron density of $\sim 200 \mathrm{~cm}^{-3}$ in 30 Dor.

\section{Variation of $[\mathrm{CII}$ emission within the LMC}

To examine the variations of $L_{[\mathrm{C} \text { II] }} / L_{\mathrm{TIR}}$, we show a ratio map of $L_{[\mathrm{C} \text { II] }} / L_{\mathrm{TIR}}$ overlaid with the boundaries of the SF regions in Fig. 3. The pixels with the lowest $L_{[\mathrm{C} \text { II] }} / L_{\mathrm{TIR}}$ are associated with the centres of the brightest SF regions, confirming the scenario in which the PE heating is least efficient at high density.

The distribution of [C II] and TIR from the different phases are summarised in Table 2. Although the SF regions have much higher values of [C II] surface brightnesses, approximately half of the $[\mathrm{CII}]$ emission originates from the diffuse medium. The SF regions contribute just less half of the [C II luminosity $(\sim 48 \%)$.

Figure 4 shows $L_{[\mathrm{CII}} / L_{\mathrm{TIR}}$ as a function of the $\mathrm{H} \alpha$ surface brightness. As can be seen, the efficiency is roughly constant across the range of $\mathrm{H} \alpha$ surface-brightnesses. There are some deviant pixels with high $L_{[\mathrm{C} \text { II] }} / L_{\mathrm{TIR}}$, in particular at the lowest values of $S_{\mathrm{H} \alpha}$, which are most-likely the result of the noise in the [C II] map. The bulk of our points clutter around $L_{[\mathrm{C} \text { II] }} / L_{\mathrm{TIR}} \simeq$ 0.005 , which is similar to the values found by Malhotra et al. (2001) and Hunter et al. (2001), who examine spiral and irregular galaxies (including the LMC). There is evidence that on the whole the 30 Dor region has a slightly lower ratio of $L_{[\mathrm{C} \text { II] }} / L_{\mathrm{TIR}}$ than the rest of the LMC by less than $10 \%$ (see also Fig. 3 ).

Theoretically, such a decrease in this ratio towards the densest regions is expected. The PDR models of Tielens \& Hollenbach (1985b,a), and Wolfire et al. (1990) show that [C II] emission levels off at the highest gas temperatures, radiation fields and gas densities. Other lines take over (part of) the cooling process at high gas temperature and high gas density. Since dust temperature roughly scales with gas temperature and gas density, it is possible that the observed decrease is associated with the critical temperature and critical density of the $\mathrm{C}^{+}$ transition being reached. We, however, do examine alternative explanations in the following sections.

\section{Distribution of $[\mathrm{CII}]$ emission and grain component emission in the LMC}

The $8,24,70$ and $160 \mu \mathrm{m}$ bands used in this study trace the emission from distinct grain populations. We use these measurements to study [C II] cooling and PE heating with respect to grain abundance. We adopt the generally accepted interpretation of the dust constituents and at which wavelength they emit (e.g. Desert et al. 1990; Draine \& Li 2001). The $8 \mu \mathrm{m}$ band is dominated by PAH emission. The $24 \mu \mathrm{m}$ band mainly traces the emission of stochastically heated carbonaceous grains with sizes less than $\sim 0.01 \mu \mathrm{m}$; termed very small grains (VSGs). The $160 \mu \mathrm{m}$ band mainly traces the grains larger than $\sim 0.01 \mu \mathrm{m}$, in radiative equilibrium; termed big grains (BGs). Finally, the emission detected in the $70 \mu \mathrm{m}$ band probably represents a combination of the BG continua and the VSG emission, and may also trace grains stochastically heated, thus not in thermal equilibrium with the interstellar radiation field. Hereafter, we represent the PAH, VSG and BG components with 8, 24 and $160 \mu \mathrm{m}$ band, respectively.

The spatial variation of PAH emission in galaxies has been studied extensively (e.g. Roche \& Aitken 1985; Aitken \& Roche 1985; Leach 1987; Voit 1992; Cesarsky et al. 1996; Verstraete et al. 2001; Siebenmorgen et al. 2004; Povich et al. 2007). The studies consistently find a lack of PAH emission for the most active regions, i.e., H II regions, starburst galaxies and AGNs. An interpretation is that the PAHs in these regions are destroyed due to the hard, intense radiation field. The VSG grain emission peaks in the HII region while the PAH emission peaks in the adjacent PDR.

We present ratio maps of $S_{8}, S_{24}, S_{70}$ and $S_{160}$ to $S_{\text {TIR }}$ in Figs. 5a-d. The grey lines enclose the SF regions as given by our $\mathrm{H} \alpha$ criterion. Consistent with the studies mentioned above, Figs. 5a,b show that the relative PAH emission is weak in the SF regions, while the VSG emission peaks in the SF regions. Figure $5 \mathrm{~d}$ shows that the $\mathrm{BG}$ emission relative to TIR mostly peaks in the diffuse medium. BGs may be present throughout the LMC, the high flux of UV photons in the H II regions entails that they are hotter in these regions and therefore radiate at wavelengths blueward of the $160 \mu \mathrm{m}$ band. Finally, we note that the $70 \mu \mathrm{m}$ emission relative to TIR (Fig. 5c) most closely follows that of the VSG emission.

\subsection{Grain component emission relative to [C II] emission}

To study how the $[\mathrm{C} \mathrm{II}]$ emission varies with grain population, we examine this parameter as a function of the ratios of grain component emission. It should be noted, though, that the ratios represent ratios of grain emission and not those of grain abundances.

Figure 6 presents $S_{[\mathrm{CII}}$ as a function of $S_{8} / S_{24}, S_{24} / S_{160}$, and $S_{8} / S_{160}$. We omit discussion of ratios with the $70 \mu \mathrm{m}$ band as they exhibit the same behaviours as the ratios with the $24 \mu \mathrm{m}$ band. We note the following trends:

- in the SF regions where the [C II] emission is highest, the $\mathrm{PAH}$ emission is low relative to the VSG emission (Fig. 6a), which is explained by the fact that the PAHs do not survive in $\mathrm{H}$ II regions where the VSGs are known to peak. As the $S_{8} / S_{24}$ ratio increases, the pixels trace more and more of the diffuse medium where the [C II] emission is the lowest;

- panel $b$ shows again that those regions with very prominent $24 \mu \mathrm{m}$ emission, i.e. SF regions, show strong [C II] emission;

- Fig. 6c shows that there is no correlation between the [C II] emission and the $S_{8} / S_{160}$ ratio for the diffuse medium. Some fraction of the SF pixels, though, show a rough 
Table 2. Contribution of [C II] from various regions in the LMC.

\begin{tabular}{|c|c|c|c|c|c|c|}
\hline Region & $L_{\left[\mathrm{C}_{\text {II] }}\right.} / L_{\left[\mathrm{C}_{\mathrm{II}}\right] \text {,total }}$ & Area/Area $a_{\text {total }}$ & $L_{\mathrm{TIR}} / L_{\mathrm{TIR}, \text { total }}$ & $L_{\left[\mathrm{C}_{\text {II] }}\right.} / L_{160}$ & $L_{\left[\mathrm{C}_{\mathrm{II}}\right]} / L_{[\mathrm{TIR}]}^{a}$ & $L_{\left[\mathrm{C}_{\mathrm{H}]}\right]} / L_{[\mathrm{FIR}]}^{b}$ \\
\hline Diffuse regions & $51.8 \pm 0.1 \%$ & $86 \%$ & $59.0 \pm 0.1 \%$ & $0.032 \pm 0.1 \%$ & $0.40 \pm 0.01 \%$ & $0.80 \pm 0.01 \%$ \\
\hline SF regions & $48.2 \pm 0.1 \%$ & $14 \%$ & $41.0 \pm 0.1 \%$ & $0.063 \pm 0.1 \%$ & $0.55 \pm 0.01 \%$ & $1.10 \pm 0.01 \%$ \\
\hline 30 Dor & $8.1 \pm 0.1 \%$ & $0.8 \%$ & $9.0 \pm 0.1 \%$ & $0.072 \pm 0.1 \%$ & $0.42 \pm 0.01 \%$ & $0.84 \pm 0.01 \%$ \\
\hline N11 & $2.2 \pm 0.1 \%$ & $0.7 \%$ & $2.0 \pm 0.1 \%$ & $0.058 \pm 0.1 \%$ & $0.57 \pm 0.01 \%$ & $1.14 \pm 0.03 \%$ \\
\hline total LMC & $100 \%$ & $100 \%$ & $100 \%$ & $0.042 \pm 0.1 \%$ & $0.46 \pm 0.01 \%$ & $0.92 \pm 0.02 \%$ \\
\hline
\end{tabular}

${ }^{a}$ Also referred to as the photoelectric efficiency (see Sect. 7.2 ). ${ }^{b}$ FIR is defined using the IRAS bands (see Sect. 2.2). TIR assumes the factor of 2 increase from the FIR, as found by Hunter et al. (2001) and discussed in the text (Sect. 2.2). Due to the calibration uncertainties of the BICE map, all of these values have a systematic uncertainty of $\pm 30 \%$. The uncertainties in the table reflect the random noise which is important for comparing the relative.

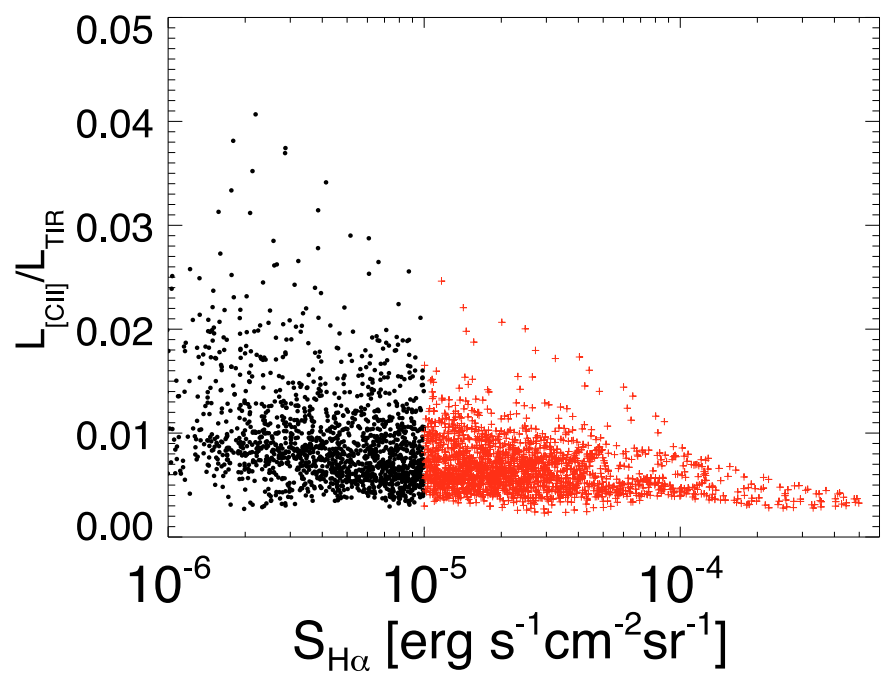

Fig. 4. $L_{\text {[C }}{ }_{\text {II }} / L_{\mathrm{TIR}}$ for each pixel plotted against its $\mathrm{H} \alpha$ surface brightness. The black dots and red crosses denote the diffuse and SF pixels respectively. (See online version for colour.)

increase with [C II] as a function of $S_{8} / S_{160}$. The regions with the most intense radiation fields will heat the surrounding grains to very hot temperatures so that the BG emission will shift to bluer wavelengths, outside of the $160 \mu \mathrm{m}$ filter. Toward the SF regions, the amount of PAH emission also decreases (see above). The decrease in the $160 \mu \mathrm{m}$ band is greater than the decrease in the PAH emission toward the most intense SF regions. This distinction between the brightest and the fainter SF regions is not reflected in the relative [C II] strength (Fig. 6e).

\subsection{Theoretical description of PE}

PE heating occurs as follows: absorption of a far-ultraviolet (FUV) photon by an interstellar dust grain liberates an electron within the grain. The electron travels through the grain, escapes, and then overcomes any Coulomb attraction if the grain is charged. If the absorbed energy exceeds the work function of the grain plus its Coulomb potential, the electron escapes with excess kinetic energy. That energy goes into heating the ISM via collisions with the gas species. Because of this Coulomb potential, PE heating efficiency is highly dependent upon the charge state of the grain. This, in turn, is dependent upon the physical conditions which determine grain ionisation and recombination rates. The PE efficiency $(\epsilon)$ is defined as:

$\epsilon \equiv \frac{\Gamma_{\mathrm{PE}}}{P_{\mathrm{abs}}}$

where $P_{\mathrm{abs}}$, represents the power absorbed by the grains and $\Gamma_{\mathrm{PE}}$ is the photoelectric heating rate. Thus, $\epsilon$ is the fraction of the power absorbed by the grains that goes into heating the ISM.

Photoelectric efficiencies as a function of environmental conditions, grain size distributions and grain compositions were calculated semi-empirically by de Jong (1977), and ab initio calculations have been made by Weingartner \& Draine (2001) and Bakes \& Tielens (1994).

Assuming an MRN grain distribution and only carbonaceous grains, Bakes \& Tielens (1994) derive an analytic expression for PE efficiency (valid for gas temperatures much less than $10000 \mathrm{~K})$ :

$\epsilon\left(G_{\mathrm{o}}, T, n_{\mathrm{e}}\right)=\frac{4.87 \times 10^{-2}}{1+4 \times 10^{-3} \gamma^{0.73}}$,

where $\gamma$ is the ratio of ionisation to recombination rate $\left(G_{\mathrm{o}} T^{1 / 2} n_{\mathrm{e}}{ }^{-1}\right)$. Thus, high ionisation rates will increase grain charge and therefore lower efficiency.

\subsection{A proxy for $\epsilon$}

$L_{[\mathrm{C} \text { II] }} / L_{\mathrm{TIR}}$ is a proxy for $\epsilon$ given several assumptions: $i$ ) that $\mathrm{PE}$ heating and the $\mathrm{C}^{+}$transition dominate the heating and cooling processes respectively, i.e. $\Gamma_{\mathrm{PE}}=\Lambda \cong L_{[\mathrm{C} I]}$ (where $\Lambda$ represents the cooling rate); and ii) and that interstellar grains and molecules re-radiate all of the energy absorbed in the infrared. However, the [C II] line is not always the dominant coolant. Therefore, to observationally estimate $\epsilon$, some authors include the measured luminosities of other FIR lines (e.g. Meixner et al. 1992; Young Owl et al. 2002). We do not have maps of the LMC in other FIR lines, and thus can not include their contribution in the cooling rate. Therefore, it should be kept in mind that our calculations of $\epsilon$ represent a lower limit on the actual values of PE efficiency, in particular in the densest regions.

Here we estimate the contribution from other lines that may be missing within the $15^{\prime}$ beam. We perform this estimation in the 30 Dor region, as this is the region in the LMC where the contributions of other FIR lines to the cooling rate should be the most significant. We estimate the contribution from the [O I] $63 \mu \mathrm{m}$ line, the dominant cooling line in regions where [C II] cooling is suppressed. The 30 Dor complex was measured in [C II] and [OI] $(63 \mu \mathrm{m})$ by Poglitsch et al. (1995) with a $55^{\prime \prime}(F W H M)$ beam aboard the KAO. They found peak 
a)
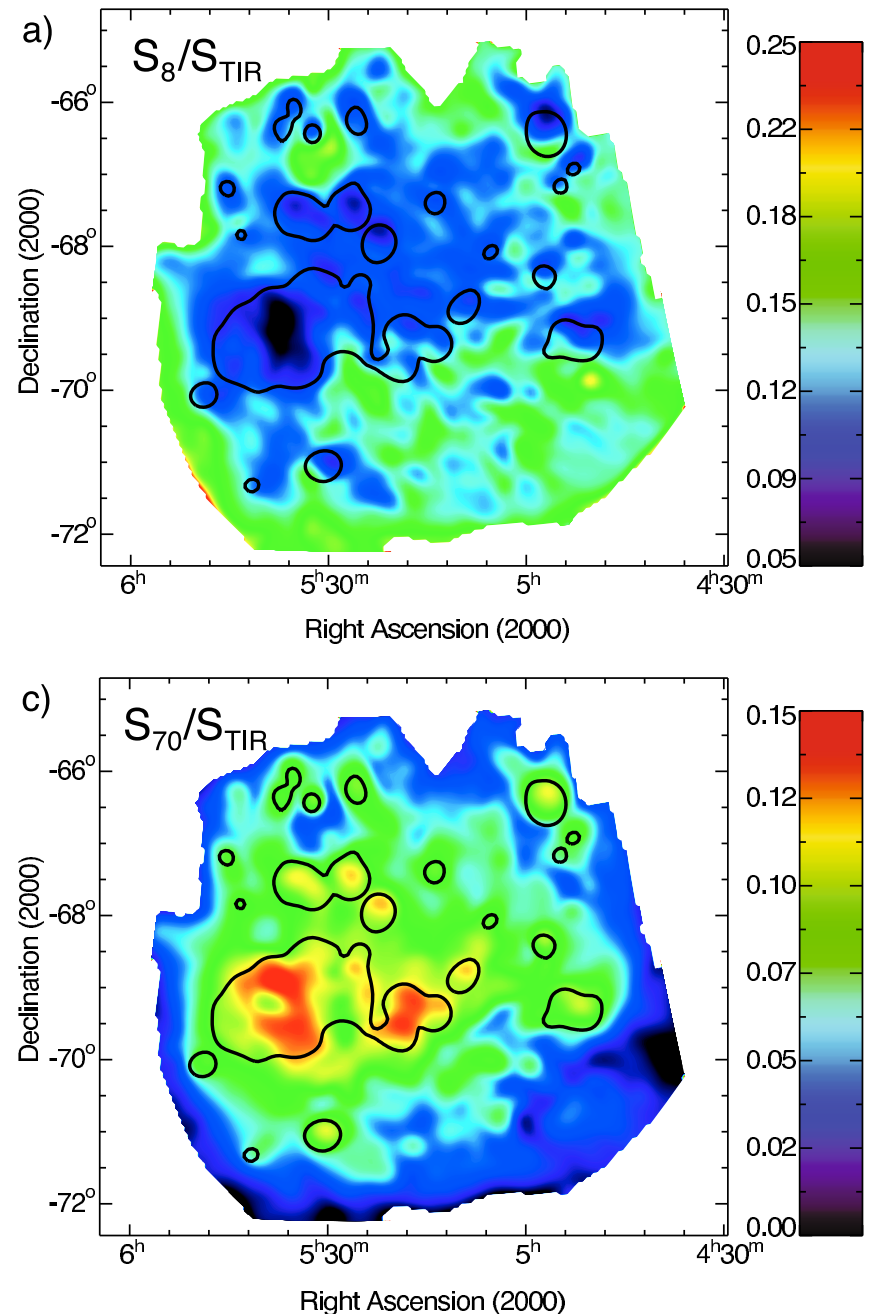
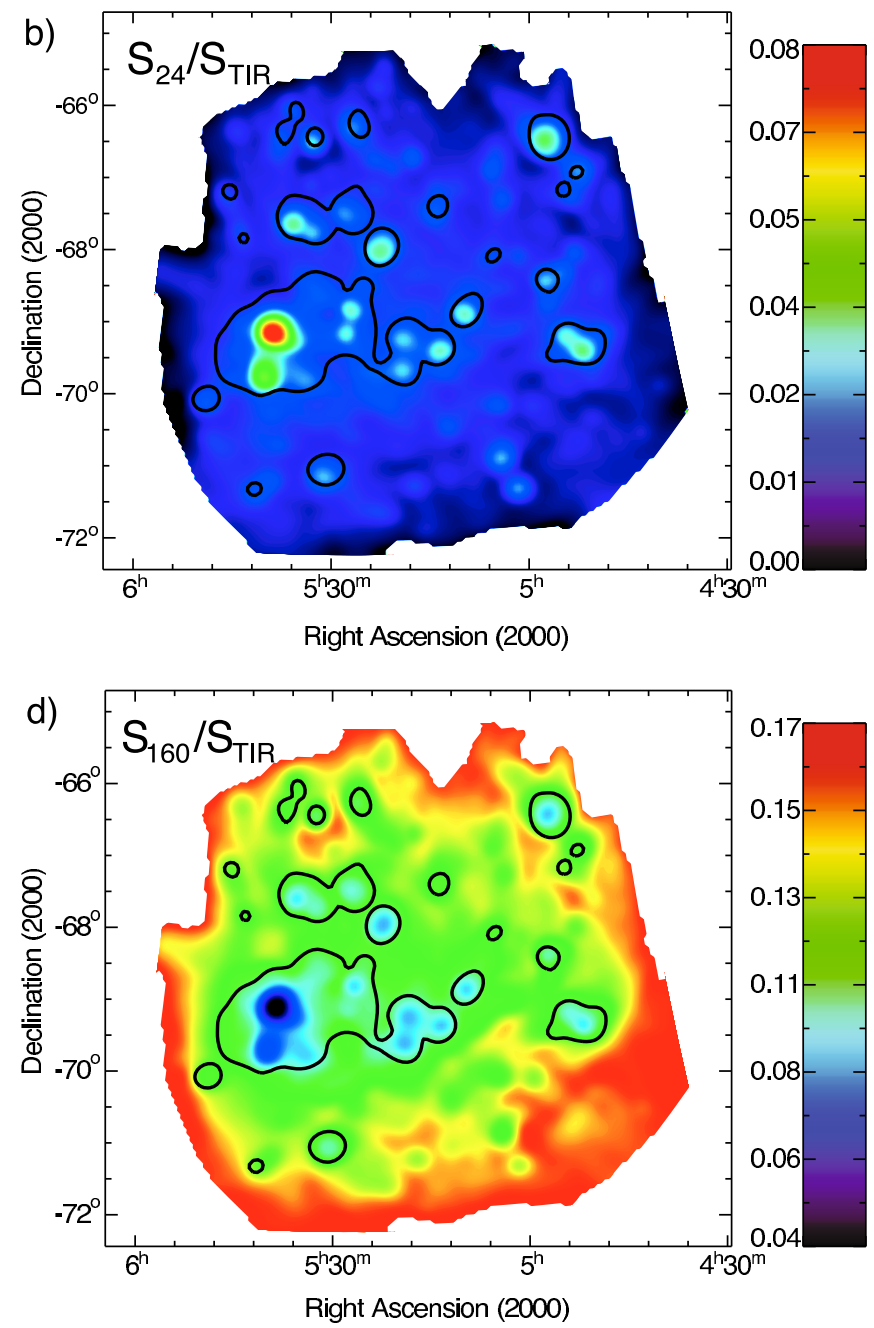

Fig. 5. Ratio maps of the 8 a), 24 b), 70 c) and $160 \mu \mathrm{m}$ d) bands to the TIR emission. Contours enclose the SF regions. (See online version for colour.)

intensities in [C II] and [O I] of $1 \times 10^{-3} \mathrm{erg} \mathrm{s}^{-1} \mathrm{~cm}^{-2} \mathrm{sr}^{-1}$ and $6.1 \times 10^{-4} \mathrm{erg} \mathrm{s}^{-1} \mathrm{~cm}^{-2} \mathrm{sr}^{-1}$ respectively. Vermeij et al. (2002a) also measured these lines for several regions in 30 Dor with the LWS which has a beam $80^{\prime \prime}$. They found that the [O I] intensity is about twice the [C II] intensity.

To estimate the contribution of [O I] in our $15^{\prime}$ beam, we assume two components within the beam: 1) the smaller region as measured by Poglitsch et al. (1995); and 2) more extended emission. For 1, we use the values as measured by Poglitsch et al. (1995). For 2, we use the differences between the line strengths as listed by Vermeij et al. (2002a) and listed by Poglitsch et al. (1995). We assume that the ratio of [O I]/[C II] in the extended region holds throughout the $15^{\prime}$ beam and scale that to the full [C II] measured in the large beam. This is a very conservative estimate of the [O I] contribution because it probably overestimates the $[\mathrm{OI}]$ line strength as the true ratio most likely decreases with distance. We thus estimate the contribution of [O I] $63 \mu \mathrm{m}$ to be $\sim 20 \%$ of the total gas cooling rate. This number should be lower in other regions in the LMC since 30 Dor represents the most extreme SF region. We conclude that, for our $15^{\prime}$ beam, the [C II] is representative of the total gas cooling rate, but may slightly underestimate the cooling rate in the most extreme cases.

Of the SF regions, 30 Dor has the lowest efficiency as it is the most intense SF region in the LMC (See Table 2). In contrast the second brightest SF region, N11, has a higher than average efficiency. The regions defining 30 Dor and N11 were chosen by constructing a rectangle centred on the brightest pixel in the $24 \mu \mathrm{m}$ image. The edges of the rectangles approximate three times the mean $S_{24}$ level of the diffuse regions. We have included the resulting rectangles in Fig. 1a. One puzzling result of this is that on average the diffuse regions exhibit a lower efficiency than do the SF region. It should be noted that most of the diffuse regions are faint and therefore affected more by the high noise in the BICE map.

\subsection{The limited sensitivity of the BICE map: validity of observed efficiency variations}

Before we can draw conclusions on the variations observed in the observed photo-electric efficiency parameter, we explored the possible existence of a systematic variation of the efficiency simply as a function of $G_{0}$ by running a set of simulations. The BICE map has limited sensitivity and as a result a large part of the diffuse medium remains undetected or has flux levels comparable to the noise level (see Fig. 3). Therefore, extra care must be taken in deriving representative values of the [C II] intensity in the diffuse medium. In these simulations we assume that $S_{\mathrm{TIR}}$ is a proxy for $G_{0}$. We simulate observed [C II] maps by applying a function of the form $\epsilon=f\left(S_{\mathrm{TIR}}\right)$. The $S_{\mathrm{TIR}}$ map is constructed using the Spitzer images and the uncertainty in this map is 

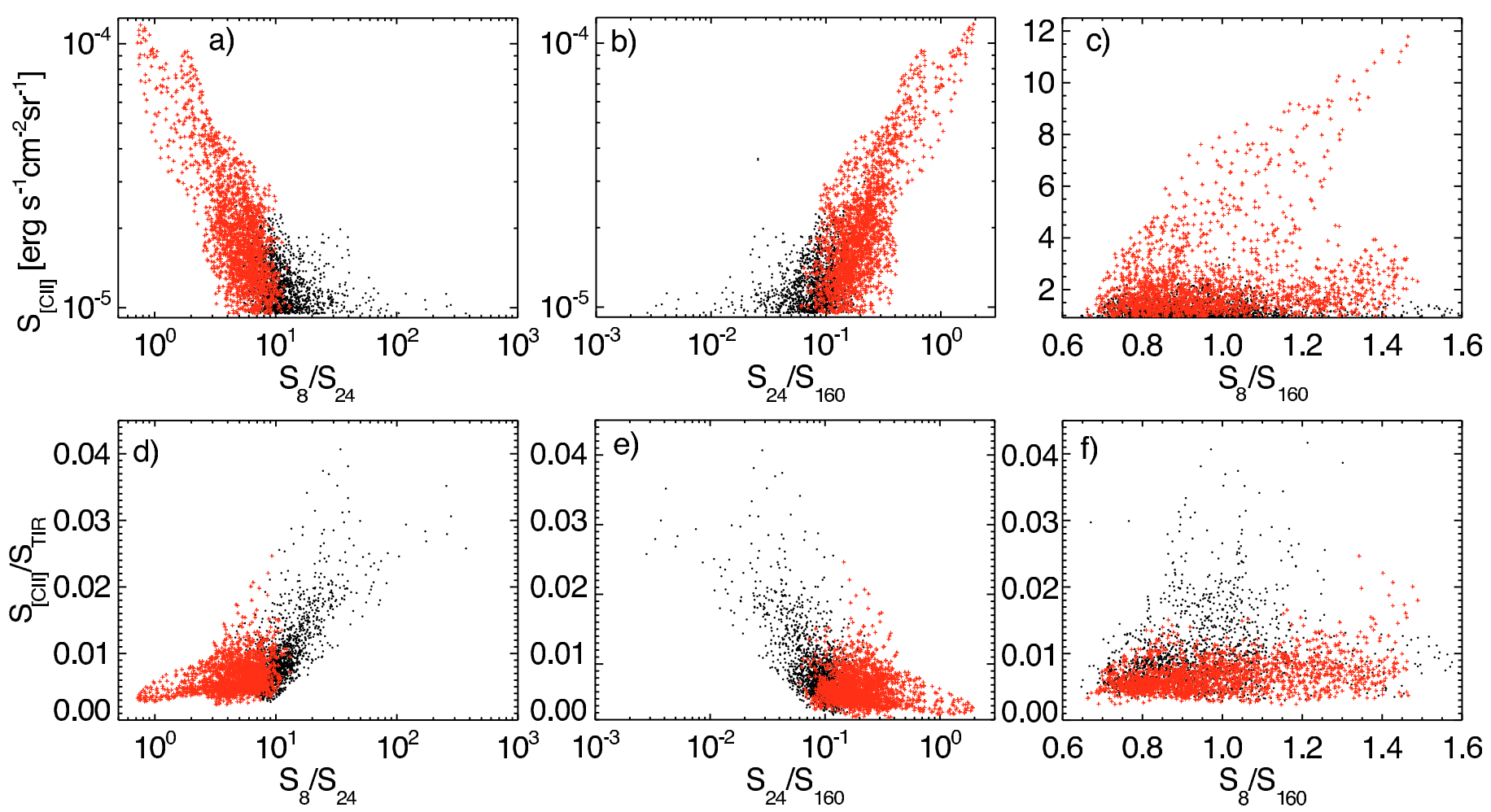

Fig. 6. [C II] surface brightness (top) and the $S_{\left[\mathrm{CII}_{\mathrm{II}}\right.} / S_{\mathrm{TIR}}$ (bottom) as a function of grain component emission ratios as traced by the Spitzer bands. The black dots and red crosses denote the different diffuse and SF pixels, respectively. Note, that the high values in the bottom three panels are due to noise in the $[\mathrm{C}$ II] map (See text for details). See online version for colour.

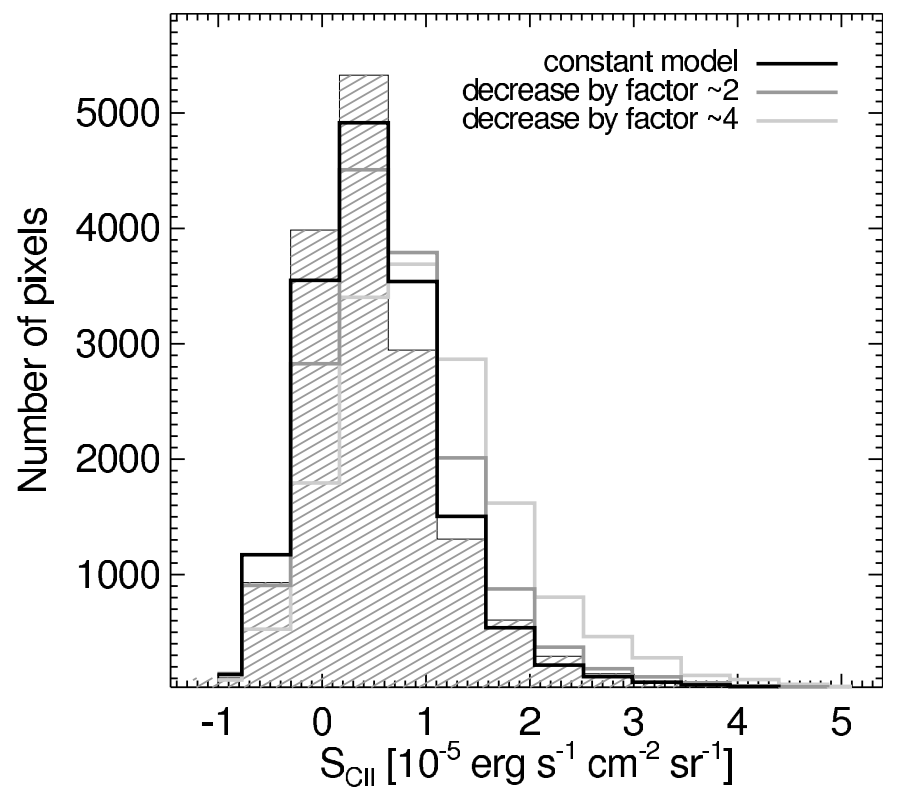

Fig. 7. Fluxes as observed in the BICE maps versus those obtained from a simple simulation (Sect. 7.4). The figure shows the observed histogram of [C II] surface-brightness (solid), the bin width is $1 \sigma$ in [C II]. The best fit model is shown in black (text for details). The dark grey line indicates the result of a simulation with a moderate decrease in efficiency as a function of TIR. The light grey line corresponds to a model with an decrease by a factor 4 , the latter simulation is clearly inconsistent with the observations as it over predicts the number the number of bright pixels.

negligible compared to the BICE map. After this we add noise according to the noise level of the BICE map. Figure 7 compares the observed histogram of [C II] values with several predicted histograms obtained following the simulation outlined above. We have simulated maps using functions of two forms: linear $f\left(S_{\mathrm{TIR}}\right)=a+b^{*} S_{\mathrm{TIR}}$ and power-law $f\left(S_{\mathrm{TIR}}\right)=a^{*} S_{\mathrm{TIR}}^{p}$. We determine the parameters which best approximate the distribution of the observed $S_{[\mathrm{CI}]}$. Some results of these simulations are shown in Fig. 7. The closest match is obtained when assuming a constant efficiency across the full range of $G_{0}$. The data do not exclude modestly higher efficiencies in the faintest regions by about a factor of two. Steeper gradients are excluded, since they clearly over-predict the number of pixels with values between 3 and $5 \sigma$ in the BICE map. At a given value of $G_{0}$ we do find a significant spread of $S_{[\mathrm{C} \text { II] }} / S_{\mathrm{TIR}}$ in our simulation. The spread, which is independent of $S_{\text {TIR }}$, has a magnitude of roughly a factor of two. The main conclusions of the simulations are as follows:

- the value of $S_{\text {[C II] }} / S_{\text {TIR }}$ is independent of $S_{\text {TIR }}$ over the range from $3 \times 10^{-4}$ to $3 \times 10^{-2} \mathrm{erg} \mathrm{s}^{-1} \mathrm{~cm}^{-2} \mathrm{sr}^{-1}$;

- the mean value of $\epsilon$ across the LMC is $0.45 \%$;

- there is a spread around this value between 0.3 and 0.6;

- there is a modestly higher value of $S_{[\mathrm{C} \text { II }} / S_{\mathrm{TIR}}$, up to $\sim 1 \%$ in the faintest regions;

- the only region exhibiting a systematically lower value is the 30 Dor region with a mean value of $S_{[\mathrm{CII}]} / S_{\mathrm{TIR}}$, of $0.35 \%$.

\section{8. [C II] emission, photoelectric efficiency and radiation field in the LMC}

Here, we examine the variations in the observed [C II] emission and PE efficiency as functions of radiation field. 


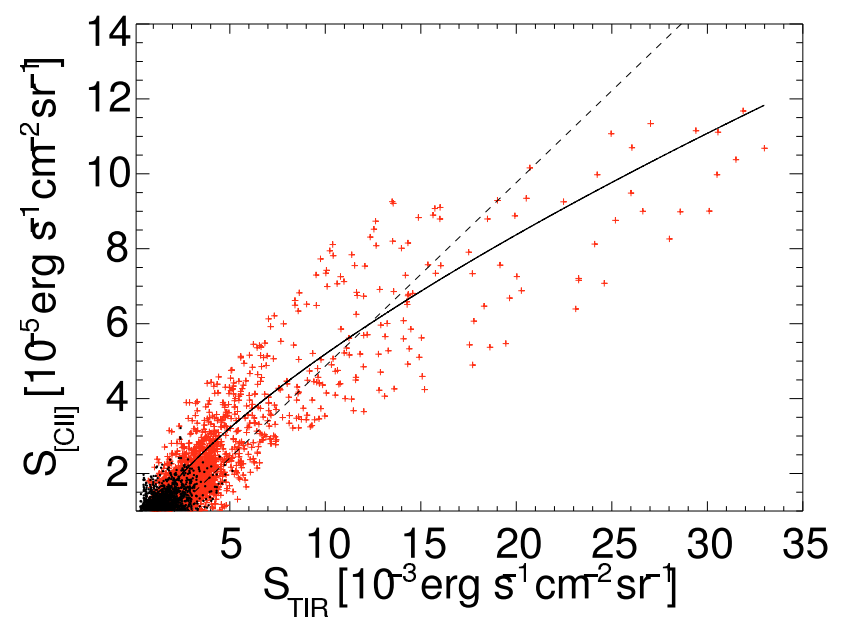

Fig. 8. $S_{[\mathrm{CI}}$ plotted as a function of $S_{\mathrm{TIR}}$ for the diffuse and SF pixels. The solid line is a power law to the data. The dashed line is a linear fit to the data with the $y$ intercept set to zero. Colours and symbols as in Fig. 4. Colours and symbols as in Fig. 4, see online version for colour.

\section{1. $S_{[\mathrm{CII}]}$ and radiation field}

We can take $S_{\text {TIR }}$ as a proxy for the UV radiation field in the case that most of the power absorbed by the dust is in the UV, and the dust radiates isotropically. We plot $S_{[\mathrm{CII}]}$ as a function of $S_{\text {TIR }}$ in Fig. 8. The [C II] emission increases as a function of $S_{\text {TIR }}$ and flattens at the highest radiation fields, which is clearly seen in the power-law fit. This flattening is dominated by the low efficiency observed in 30 Dor. We also tried to fit a straight line to the data with the $y$ intercept set at zero. The data is clearly better described by the power law. The difference between the power-law and the linear fit at the highest values of $S_{\text {TIR }}$ is $30 \%$, which is more than we can comfortably explain by missing line emission from other cooling fine structure lines (Sect. 7.3). The power-law fit yields the following prescription for the [C II] surface brightness as a function of total infrared surface brightness throughout the LMC:

$S_{\text {[C II] }}=1.25 \times 10^{-3} S_{\mathrm{TIR}}^{0.69}$,

where surface brightness is given in erg $\mathrm{s}^{-1} \mathrm{~cm}^{-2} \mathrm{sr}^{-1}$. The equation is valid for $S_{\mathrm{TIR}}$ between 3.2 and $33 \times 10^{-4} \mathrm{erg} \mathrm{s}^{-1} \mathrm{~cm}^{-2} \mathrm{sr}^{-1}$, the range from which the fit was made.

We interpret the flattening at the highest TIR in Fig. 8 as a decrease in the PE heating rate. In high radiation field and high temperature environments, grain charging effects become important, the PE heating efficiency decreases and thus the line cooling drops. This flattening is also observed in KAO data of Galactic and extragalactic regions by Stacey et al. (1991). In Fig. 17 of their paper, they plot $S_{[\mathrm{C} \text { II] }}$ as a function of the FIR surface brightness (which they call $\chi_{\text {FIR }}$ ) normalised to $2 \times 10^{-4} \mathrm{erg} \mathrm{s}^{-1} \mathrm{~cm}^{-2} \mathrm{sr}^{-1}$. We show our data, their data and Eq. (4) in Fig. 9. We have converted our TIR values into $\chi_{\text {FIR }}$ by assuming a factor of two for TIR to FIR as given by Hunter et al. (2001). We also plot tracks of constant PE efficiency in Fig. 9 (dashed lines) from $\epsilon=5 \%$ (left) to $5 \times 10^{-3} \%$ (right); where $\epsilon=5 \%$ is chosen because it is close to the highest efficiency in the theory of Bakes \& Tielens (1994).

Our data throughout the LMC follow the trend of Stacey et al. (1991) and extends to the lower left portion of the plot. To understand why our SF points do not occupy the upper right portion of the graph, one must consider that our beam size ( $\sim 200$ pc) undoubtedly entails considerable mixing of the phases of the ISM. Stacey et al. (1991) include measurements of 30 Dor (indicated on Fig. 9). We use this to gauge how beam size affects this figure. The measurement of 30 Dor from our data is indicated with a star. 30 Dor in the BICE beam has significantly lower IR and [C II] surface brightnesses, by factors of about 125 and 15 respectively. Note, that because of the much smaller beam of the $\mathrm{KAO}$, and because the Galactic regions are closer, the data of Stacey et al. (1991) probe much smaller spatial scales. On these scales, the contribution to cooling from the [OI] line as compared to the $[\mathrm{C} \mathrm{II}]$ line might be important. If the [O I] line were included in the Stacey et al. (1991) data, the points at the upper-right side of the diagram would move up. Even so, inclusion of these other lines would most likely not be sufficient to move the data points up to the efficiency of the LMC, as this would require them to be moved up the $y$-axis by at least an order of magnitude. Such cooling contributions from other lines, even in the most intense regions, are not expected. As with the decreasing trend of efficiency in the Stacey data, we note that for our data, 30 Dor shows the most prominent decrease in efficiency. Perhaps this reflects the fact that 30 Dor is so bright that it dominates the emission even in the large beam.

One must note though, that the other variables which control the PE heating (i.e. gas temperature and electron density) are not constant throughout Figs. 8 and 9. Perhaps the considerable spread in [C II] at any given $S_{\text {TIR }}$ in Fig. 8 and can be attributed to those other variables.

\section{Correlation between radiation field and electron density}

In this section we use the data and the physics of PE heating to derive values for $G_{0}$ and $n_{\mathrm{e}}$. We find that the observed constant efficiency translates into a tight correlation between the density of the radiation field and the density of electrons. We discuss the physical interpretation of this correlation and in particular we compare our results with those of Young Owl et al. (2002) and Malhotra et al. (2001).

To first order the observed efficiency is constant throughout the LMC. This constancy of $0.45 \%$ translates into a constant value of $\gamma \approx 4 \times 10^{4}$ (Eq. (3)). A typical value for PDR gas temperature is $T \sim 300 \mathrm{~K}$. To account for the contribution from the diffuse ISM ( $T \sim 50-100 \mathrm{~K} 2005)$ we adopt a value of $T=75 \mathrm{~K}$. Assuming an average temperature of $75 \mathrm{~K}$ across the $\mathrm{SF}$ pixels we find that all of the LMC regions, averaged over our 225 pc regions here, are typified by $G_{0} / n_{\mathrm{e}} \approx 5000$.

Several explanations for the constancy of the observed efficiency and, as a consequence, the derived interrelation between $G_{0}$ and $n_{\mathrm{e}}$ come to mind.

i) If, in our large beam, the radiation field is dominated by similar PDR regions, then the number of PDRs in that beam will determine variations in the total radiation field, but will not change the intrinsic PE efficiency.

- ii) If, in our large beam, the light is dominated by emission from the diffuse medium, PE efficiency should remain fairly constant as the physical conditions within this medium are relatively invariant.

- iii) The value of $G_{\mathrm{o}} / n_{\mathrm{e}}$ is constant throughout the different types of media that make up the LMC. In fact, for several Galactic PDR regions, Young Owl et al. (2002) do indeed find that $G_{\mathrm{o}}$ and $n_{\mathrm{e}}$ scale with each other. They studied the PDRs of a sample of reflection nebulae. Their observations of FIR atomic fine structure lines and the FIR continuum 


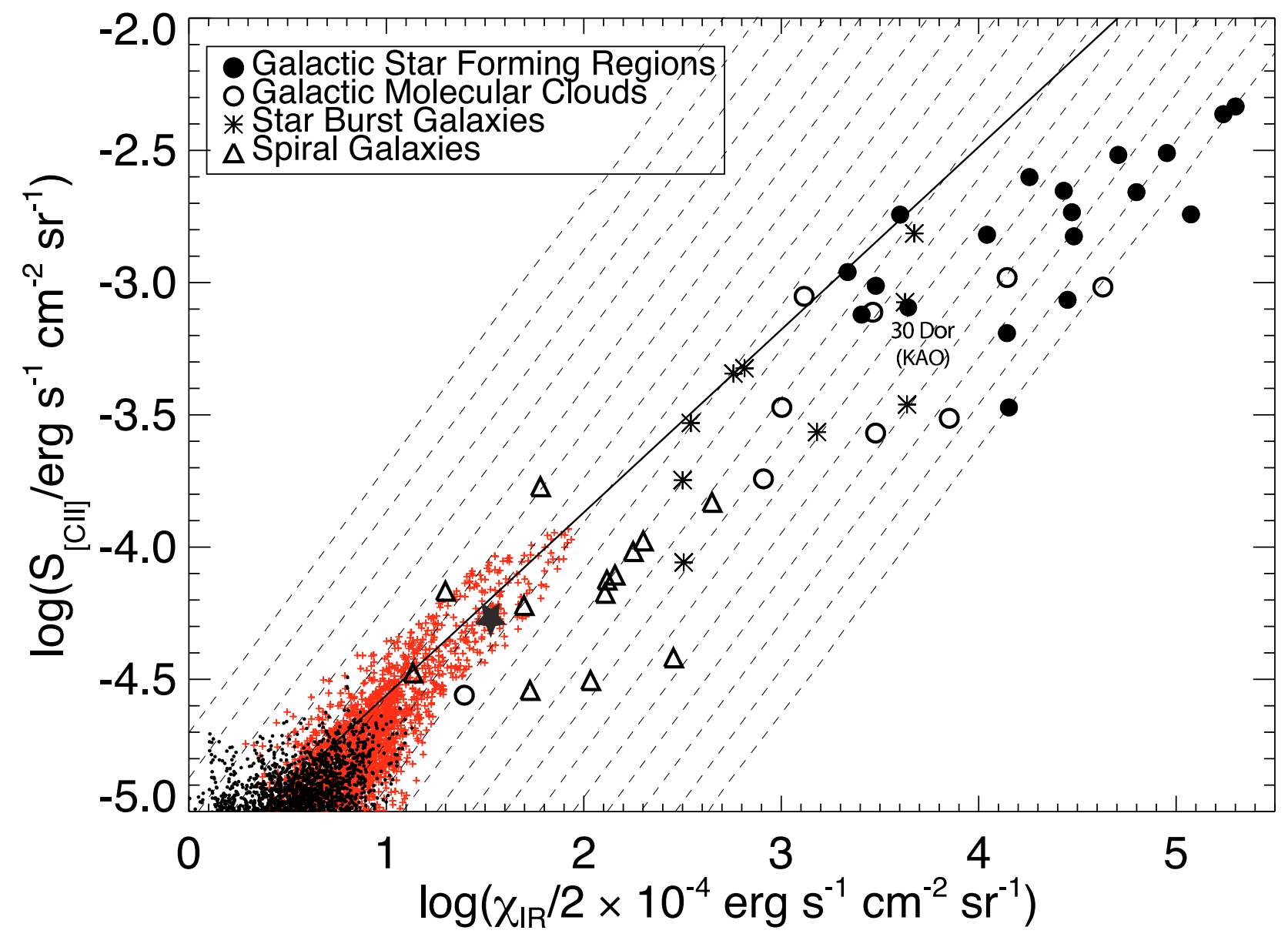

Fig. 9. Diffuse and SF data plotted over Fig. 17 from Stacey et al. (1991). The solid line is a power law fit to our LMC data. Tracks of constant PE efficiency are also plotted (dashed lines). The first track on the left has an efficiency of $5 \%$ and the last track on the right has an efficiency of $5 \times 10^{-3} \%$. The interval between each line is a step of 1.5. Colours and symbols as in Fig. 4, see online version for colour.

allowed them to obtain estimates of radiation fields and gas densities $\left(n_{0}\right)$.

It is interesting to compare our results in more detail with those obtained by Young Owl et al. (2002) and Malhotra et al. (2001). The latter measured line emission ratios across entire galaxies for a large sample of galaxies with varying morphologies. They used PDR models to obtain values for radiation fields and gas densities. Both then fitted power laws to their data. For a function of the form, $G_{\mathrm{o}}=\xi^{\prime} n_{\mathrm{o}}^{p}$, Young Owl et al. (2002) find $\xi^{\prime}=0.09$ and $p=1.2$, and Malhotra et al. (2001) $\xi^{\prime}=0.23$ and $p=1.33$ (estimated from Fig. 11 of Malhotra et al. 2001). In other words, they also find that the density scales roughly linearly with the radiation density.

Here, we estimate $G_{0}$ and $n_{\mathrm{e}}$ for independent pixels in the LC. We do this only for the SF-regions. One, because these are the brightest, reliably detected regions and two, because the conversion from $S_{\mathrm{TIR}}$ to $G_{0}\left(C_{S \rightarrow G}\right)$ uses the assumption of central illumination which is more likely to hold in those regions. We use the observed efficiency to invert the efficiency equation of Bakes \& Tielens (1994, Eq. (3)). This yields values of $\gamma$ for each pixel. We measure the $G_{0}$ using $S_{\text {TIR }}$, and assume a reasonable value for $T$. Thus we can derive values of $n_{\mathrm{e}}$ for each pixel.

Following the examples of Meixner et al. (1992), Young Owl et al. (2002), and Steiman-Cameron et al. (1997) we use the measured TIR surfaces brightnesses and assume a certain geometry in order to estimate values of $G_{0}$. Assuming 1) that the illuminating sources are at the centre of each pixel; 2) that the dust resides

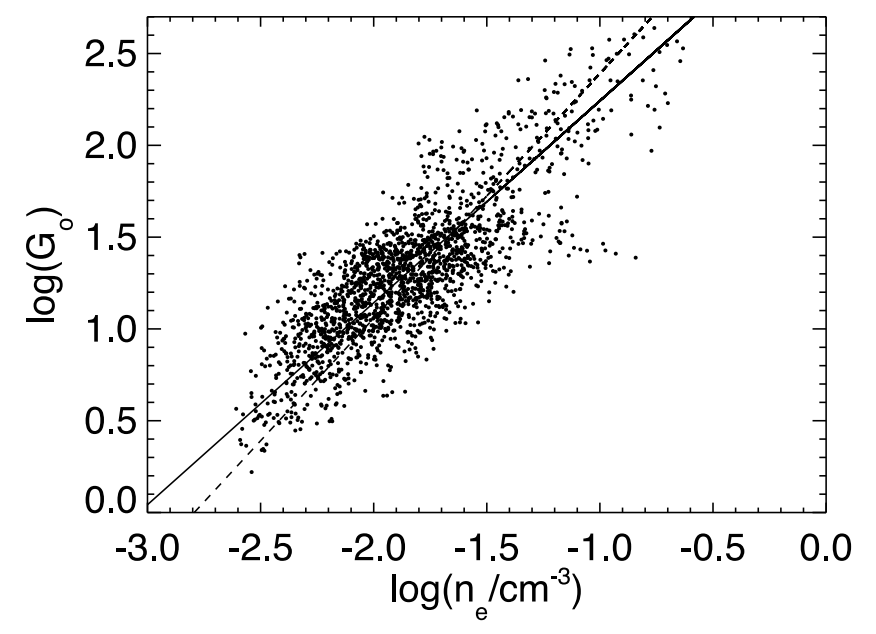

Fig. 10. $G_{\mathrm{o}}$ as a function of $n_{\mathrm{e}}$ for the SF pixels as estimated from Eqs. (3) and (5). The solid and dashed lines are fits of the equation $G_{\mathrm{o}}=\xi n_{\mathrm{e}}^{p}$. The solid line is fit with both $\xi$ and $\mathrm{p}$ as free parameters and results in $\xi=2200$ and $p=1.1$. The dashed line is a fit with $p$ set to $4 / 3$ and $\xi$ as a free variable. The result is $\xi=5300$.

at edges of the pixels; 3 ) 100\% conversion of FUV radiation to IR radiation; 4) and that the dust radiates isotropically, then

$G_{\mathrm{o}}=C_{S \rightarrow G} S_{\mathrm{TIR}}$,

with $C_{S \rightarrow G}=4 \times 10^{-4}, G_{\mathrm{o}}$ in units of the one dimensional habing field $\left(1.6 \times 10^{-3} \mathrm{erg} \mathrm{s}^{-1} \mathrm{~cm}^{-2}\right)$ and $S_{\mathrm{TIR}}$ in $\mathrm{erg} \mathrm{s}^{-1} \mathrm{~cm}^{-2}$. 


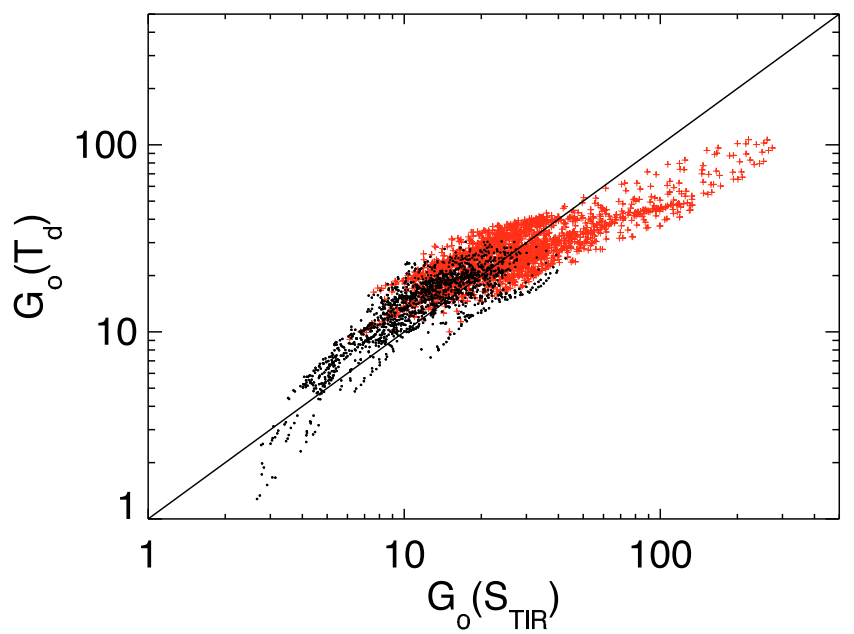

Fig. 11. Values of $G_{0}$ for each pixel derived from the dust temperature assuming graphitic grains for the dust composition versus values of $G_{\mathrm{o}}$ given by Eq. (5). The solid line denotes agreement between the two methods. Colours and symbols as in Fig. 4, see online version for colour.

Other geometries; like the dust residing halfway between the source and the edge, homogeneous distribution of sources and dust or uniformly illuminated dust; changes $C_{S \rightarrow G}$ only by a small amount. The $G_{0}$ thus calculated is shown in Fig. 10 versus the derived electron density. We find a best fit power-law of the form $G_{0}\left[\right.$ habing] $=2200 n_{\mathrm{e}}{ }^{1.1}\left[\mathrm{~cm}^{-3}\right]$. Note, that the results of Malhotra et al. (2001) and Young Owl et al. (2002) are expressed in $n_{\mathrm{o}}$ while we use $n_{\mathrm{e}}$ and thus they differ by the ionisation fraction.

In order to numerically compare our result we convert $n_{\mathrm{e}}$ to $n_{0}$ assuming that the average electron density is dominated by diffuse ISM, outside of the $\mathrm{H}$ II regions. In this case the prime donor of free electrons is carbon which is the most abundant element with an ionisation potential $(11.3 \mathrm{eV})$ below that of hydrogen $(13.6 \mathrm{eV})$. Taking into account the metallicity of the LMC we find a conversion factor $(\mathrm{H} / \mathrm{C})$ from $n_{\mathrm{e}}$ to $n_{0}$ of 6000 . It can be seen in Fig. 12 that our values follow the trend established by Young Owl et al. (2002) well.

Young Owl et al. (2002) and Malhotra et al. (2001) propose that the correlation can be physically interpreted as a balance of gas pressures between the PDR and the H II region. Young Owl et al. (2002) present a simple analytical model assuming this balance of pressures and they find that $G_{\mathrm{o}}$ should scale with $n_{\mathrm{o}}^{4 / 3}$. For both Young Owl et al. (2002) and Malhotra et al. (2001), the power of $4 / 3$ fits to within the uncertainties of their data and fits within the envelope of the uncertainty of our data (Fig. 10). We have performed an F-test to determine whether the fit with $p$ as a free parameter is an improvement compared to strict pressure balance (See Fig. 10). The shallower power-law ( $p=1.1)$ does decrease the reduced $\chi^{2}$ significantly although it remains well above unity.

The cause behind the correlation is unclear, especially one that would hold over such a range of scales. It is difficult to envisage pressure-balance between the powering $\mathrm{H}$ II regions and the $200 \mathrm{pc}$ regions of space that we are sampling. This is even more true for the results of Malhotra et al. (2001), who sample entire galaxies and still find a strong correlation between the average radiation field and the average density. Such a pressure balance would work if each single region or galaxy is dominated by a single (or at most e few) cluster(s) of young stars that cause a single prominent PDR to plough into the containing molecular cloud.

\subsection{Dust temperature in the LMC}

In the previous sections, we used the measured TIR surface brightness as a proxy for radiation field. There could be a worry, though, that $S_{\text {TIR }}$ can vary without actual variations in the radiation field. This may be due to differences in the amount of and characteristics - emitting material along the line of sight as a result of varying densities or a varying thickness of the LMC. We explore these concerns by comparing $G_{\mathrm{o}}$ as derived from $S_{\mathrm{TIR}}$ to $G_{\mathrm{o}}$ as derived by a rough calculation of the dust temperature as measured by the $70 \mu \mathrm{m}$ to $160 \mu \mathrm{m}$ ratio. This ratio in not affected by varying amounts of material along the line-of-sight.

We derive a dust temperature indicator assuming modified black-body radiation with an emissivity of the following functional form: $Q \propto v^{\beta}$, with $\beta=1.5$. This is a crude approximation, since in reality dust grains do not emit as modified blackbodies. The dust temperature will vary with grain-size and also with grain composition. Moreover, a part of the emission we observe at $70 \mu \mathrm{m}$ is not due to dust at an equilibrium temperature (See Sect. 7), but arises from stochastically heated grains. For these reasons, the absolute value of the derived temperature is not very reliable, but the relative temperatures are. In spite of the above caveats, the temperatures that we find are all-together reasonable and compare well with the results of more in-depth studies. The mean dust temperature we find $\left(\overline{T_{\mathrm{d}}}=24.0 \pm 2.2 \mathrm{~K}\right)$ is consistent with the ones found by Sakon et al. (2006, 23.9 K) and Aguirre et al. $(2003,25.0 \pm 1.8 \mathrm{~K})$.

Using the relationship between $G_{\mathrm{o}}$ and $\mathrm{T}_{\mathrm{d}}$ given by Tielens (2005) for graphite grains, we calculate $G_{\mathrm{o}}\left(T_{\mathrm{d}}\right)$ for each pixel. We plot $G_{\mathrm{o}}\left(S_{\mathrm{TIR}}\right)$ versus $G_{\mathrm{o}}\left(T_{\mathrm{d}}\right)$ for each pixel in Fig. 11 . The solid line denotes perfect correspondence. The correspondence between the two $G_{0}$ indicators is clear and thus we conclude that $S_{\text {TIR }}$ is indeed a valid proxy for $G_{0}$.

\section{Photoelectric efficiency and grain distribution in the LMC}

Theoretical studies have shown that the extent of grain charging and therefore the efficiency of PE heating is not only dependent on environmental conditions, but also on grain size and grain species. Interstellar molecules such as PAHs and the smallest grains contribute most extensively to the PE heating (Watson 1972; Bakes \& Tielens 1994; Weingartner \& Draine 2001). For example, Bakes \& Tielens (1994) have found that approximately half of the heating is from grains with less than about 1500 C-atoms $(\sim 15 \AA)$.

Qualitatively, the scaling of efficiency with grain size is due to the fact that the ionisation rate is approximately $\propto N_{\mathrm{C}}$ since the FUV absorption cross section is approximately $\propto N_{\mathrm{C}}$. But, the recombination rate increases more slowly with the number of carbon atoms as it scales with the grain size (about $N_{\mathrm{C}}^{1 / 3}$ ). Therefore, the fraction of ionised grains increases with grain size, scaling by about $N_{\mathrm{C}}^{2 / 3}$.

There has been a plethora of theoretical studies on grain species and PE heating, but relatively few observationally based studies. Therefore, we now examine PE efficiency as a function of grain species by using each band as a tracer of grain abundance.

\section{A calculation of efficiencies}

Authors usually take [C II]/FIR as a general efficiency, encompassing the contribution to PE heating of all constituent grain 
Table 3. Results of efficiency calculations.

\begin{tabular}{lccccc}
\hline \hline \multicolumn{1}{c}{ Region } & $\epsilon_{8}$ & $\epsilon_{24}$ & $\epsilon_{70}$ & $\epsilon_{160}$ & $\chi^{2}$ \\
\hline Integrated over LMC & 0.02 & 0.00 & 0.02 & 0.01 & 1.1 \\
SF regions & 0.06 & 0.00 & 0.00 & 0.01 & 2.0 \\
30 Dor & 0.05 & 0.00 & 0.00 & 0.01 & 4.7 \\
N11 & 0.05 & 0.04 & 0.00 & 0.00 & 2.8 \\
\hline Diffuse regions & 0.00 & 0.11 & 0.02 & 0.02 & 0.8 \\
\hline
\end{tabular}

populations. But, as each grain component is expected to have different intrinsic PE efficiencies, it is interesting to isolate the contribution of PE heating from the various species. Therefore, to quantify the importance of the various grain species to the PE heating process, we follow the example of Habart et al. (2001).

Using IRAS data, Habart et al. (2001) quantified the amount of emission attributed to the PAH, VSG and BG populations in the $\rho$ Opiuchi complex. Using ISO observations of the [C II], $[\mathrm{O} \mathrm{I}]$ and $\mathrm{H}_{2}$ lines, they then calculated $\epsilon_{\mathrm{PAH}}, \epsilon_{\mathrm{VSG}}, \epsilon_{\mathrm{BG}}$ with a linear combination fit of their gas cooling rate to their grain emission rates (further detail given below). They found that the PAH population is attributed with to highest photoelectric efficiency, while the BG population is attributed to the lowest. They found $\left(\epsilon_{\mathrm{PAH}}=3 \%, \epsilon_{\mathrm{VSG}}=1 \%, \epsilon_{\mathrm{BG}}=0.1 \%\right)$.

To perform this calculation, we start with the definition of efficiency for a certain grain population, $j$, which is given as

$\epsilon_{\mathrm{pop} j} \equiv \frac{\Gamma_{\mathrm{PE}}(\operatorname{pop} j)}{P_{\mathrm{abs}}(\operatorname{pop} j)} \cong \frac{S_{[\mathrm{C} \mathrm{II}]}(\operatorname{pop} j)}{S_{\text {emitted }}(\operatorname{pop} j)}$.

The substitutions of $\Gamma_{\mathrm{PE}}$ and $P_{\mathrm{abs}}$ with $S_{\text {[C II] }}$ and $S_{\text {emitted }}$ were already discussed in Sect. 7.3. To solve for the total amount of emitted [C II], we multiply both sides of Eq. (6) with the denominator on the right side of the equation, and then sum over all grain populations:

$S_{\text {[C II] }}=\sum_{\text {all pop }} \epsilon_{\text {pop j }} S_{\text {emitted }}($ pop $j)$.

Finally, if we consider the emission in the 8, 24, 70 and $160 \mu \mathrm{m}$ bands as arising from distinct grain populations, and that these are the main populations that contribute to PE heating, we obtain:

$S_{\text {[C II }]} \cong \epsilon_{8} S_{8}+\epsilon_{24} S_{24}+\epsilon_{70} S_{70}+\epsilon_{160} S_{160}$.

The variable, $\epsilon_{8}$, should reflect the of PAH efficiency, $\epsilon_{24}$ of the VSG efficiency, $\epsilon_{70}$ of the a combination of the VSG and BG efficiency and $\epsilon_{160}$ of just the BG efficiency.

To solve for the efficiencies of Eq. (8), we fit a linear combination of the IRAC and MIPS data to the [C II] map with a $\chi^{2}$ minimisation. Further, we force the efficiencies to be between 0 and 1 . We perform this calculation across the whole galaxy, for the 30 Dor and N11 regions and for the diffuse and $\mathrm{SF}$ regions in the LMC. All calculated efficiencies, along with the $\chi^{2}$ value for each fit are given in Table 3 .

Table 3 shows that with the exception of N11, $\epsilon_{24}$ and $\epsilon_{70}$ are always zero and $\epsilon_{8}$ and $\epsilon_{160}$ are non-zero. We show the values derived for the diffuse medium as well. However, these values should be taken with extreme care, because of large contribution of noise to these pixels. We have experimented with deriving grain-efficiencies also using simulated [C II] maps and find that the $2 \%$ value for the BG is found persistently but that the $\epsilon$-VSG grain is very sensitive to the exact noise characteristics of the [C II] map and can not be trusted.

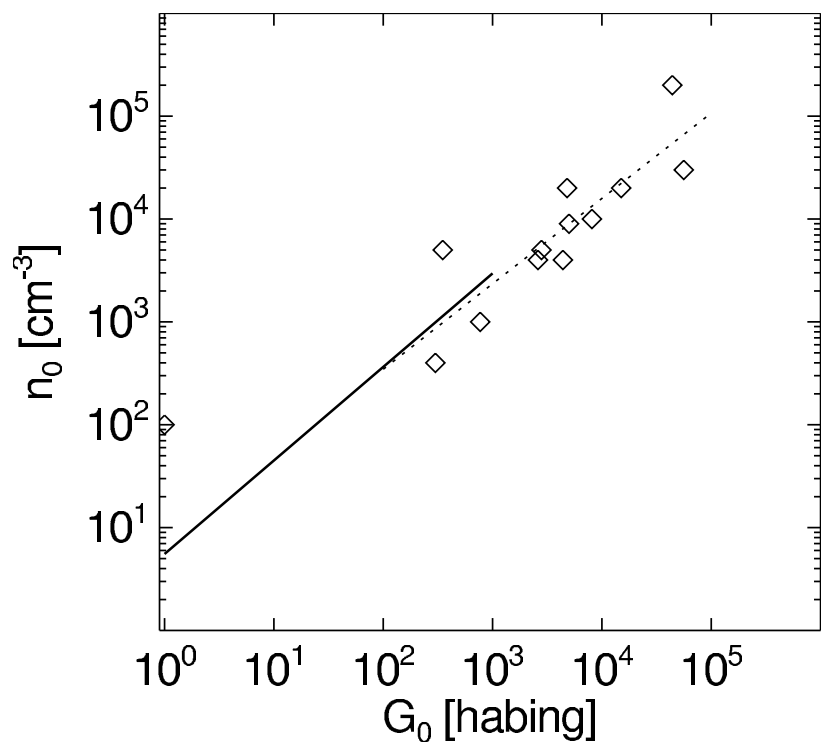

Fig. 12. Comparison of the interrelation between $G_{0}$ and $n_{0}$ that we find and that is found within galactic PDR regions by Young Owl et al. (2002). The diamonds indicate the data from Young Owl et al. (2002), while the dashed line is their best fit. The drawn line shows $n_{0}$ as a function of $G_{0}$ for each fixel in our analysis. Here $n_{0}$ is derived from $n_{\mathrm{e}}$ (Fig. 10) assuming that the electron density equals the carbon density.

The values in Table 3 show that $\epsilon_{8}$ is greater than $\epsilon_{160}$ for every region considered. This is quantitative proof that the PAH emission is more spatially correlated with the [C II] emission than the BG emission. This supports the interpretation put forth in the previous section, that the $\mathrm{PAH}$ population dominates the PE efficiency and plays an important role in the photoelectric heating of the gas.

The results for N11 obviously differ from the other regions considered as $\epsilon_{160}=0$ and as and $\epsilon_{24} \neq 0$. This might reflect offsets between the [C II] emission and the Spitzer bands due to the distinctive asymmetry towards N11 already discussed in Sect. 3 .

\section{Conclusion}

Using the MIR to FIR SAGE maps, and the BICE [C II] map of the LMC, we have, for the first time, conducted an observational study of PE heating and [C II] cooling in relation to spatially resolved grain emission throughout the LMC.

Integrated throughout the entire $\mathrm{LMC}$, the $[\mathrm{CII}]$ line accounts for $0.64 \pm 0.01 \%$ of the total infrared $(\sim 1.2 \%$ of the FIR). Applying a correction for the pixels below $2 \sigma_{[\mathrm{C} \text { II] }}$, we find that the [C II] line accounts for $1.32 \pm 0.01 \%$ of the FIR. This value is greater than that of normal and gas rich galaxies (with values typically from $0.1-1 \%$ ), as found in other low metallicity galaxies.

Distinguishing environments by $\mathrm{H} \alpha$ surface brightnesses and by location, we find that the $\left[\mathrm{C}_{\mathrm{II}}\right]$ line contributes significantly less to the TIR emission in SF regions versus diffuse ISM regions:

- $L_{[\mathrm{C} \text { II] }} / L_{\mathrm{TIR}}=0.57 \pm 0.01 \%$ for the SF regions;

- $L_{[\mathrm{C} \text { II }]} / L_{\mathrm{TIR}}=0.42 \pm 0.01 \%$ for 30 Doradus;

- $L_{[\mathrm{C} \text { II] }} / L_{\mathrm{TIR}}=0.56 \pm 0.03 \%$ for N11;

- $L_{[\mathrm{C} \text { II] }} / L_{\mathrm{TIR}}=0.79 \pm 0.01 \%$ for the diffuse regions.

We also calculate the contribution of the total output of [C II] emission from the LMC from the same regions. We find 
that, although the SF regions have the highest surface brightness values, most of the LMC's [C II] emission originates from the diffuse medium:

$$
\begin{aligned}
& -L_{[\mathrm{C} \text { II] }} / L_{\left[\mathrm{C}_{\mathrm{II}}\right] \mathrm{LMC}}=31.5 \pm 0.1 \% \text { for the SF regions; } \\
& -L_{[\mathrm{C} \text { II] }} / L_{\left[\mathrm{C}_{\mathrm{II}} \mathrm{LMC}\right.}=5.58 \pm 0.1 \% \text { for } 30 \text { Doradus; } \\
& -L_{\left[\mathrm{C}_{\mathrm{II}}\right]} / L_{\left[\mathrm{C}_{\mathrm{II}} \mathrm{LMC}\right.}=1.53 \pm 0.1 \% \text { for } \mathrm{N} 11 \\
& -L_{\left[\mathrm{C}_{\mathrm{II}}\right]} / L_{\left[\mathrm{C}_{\mathrm{II}}\right] \mathrm{LMC}}=68.5 \pm 0.1 \% \text { for the diffuse regions. }
\end{aligned}
$$

To examine variations in PE efficiency within the LMC, we use $S_{\left[\mathrm{C}_{\text {II }}\right]}$ as a proxy for the total PE heating rate. We estimate that this assumption is valid for our $15^{\prime}$ beam for all but the most active $\mathrm{SF}$ regions which might underestimate the total heating rate by at most $20 \%$. We study how PE efficiency varies with environment using the observed values of $S_{\text {TIR }}$ as an indicator of the local radiation field. As a function of $S_{\mathrm{TIR}}, S_{[\mathrm{C} \text { II] }}$ flattens at the highest value of $S_{\text {TIR }}$. The flattening trend is interpreted as a decrease in the PE efficiency for the most illuminated regions in the LMC. We provide a prescription for $S_{\text {[C II] }}$ as a function of $S_{\text {TIR }}$ (Eq. (4)) by fitting a power law to the data. Such a decrease in efficiency is theoretically expected due to grain charging effects.

Previous studies have found a correlation between these two parameters, with $G_{\mathrm{o}} \propto n_{\mathrm{e}}^{4 / 3}$. This relation has been explained by invoking a simple model assuming pressure-balance between H II regions and the adjacent PDRs. Theoretically, the PE efficiency depends strongly on the recombination-rate, and thus on the ratio of $G_{\mathrm{o}} / n_{\mathrm{e}}$. We thus calculate values for $G_{\mathrm{o}}$ and $n_{\mathrm{e}}$ using the observed efficiencies. We convert the observed $S_{\text {TIR }}$ to $G_{0}$, assuming illumination by a central source in each $45 \times 45 \mathrm{pc}^{2}$ pixel. We find that a similar scaling-relation between $G_{\mathrm{o}}$ and $n_{\mathrm{e}}$ holds for the LMC. It is unclear why the Strömgren sphere argument should hold on the large $\sim 45 \mathrm{pc}$ scale that we probe.

We analyse observed PE efficiencies in relation to the grain component emission from each Spitzer band. We note that this is the first such analysis utilising spatially resolved grain emission components throughout an entire galaxy. From the correlations between observed efficiency and the grain component emission, and from a calculation of the PE efficiencies for each population, we show that the PAH emission is the most spatially correlated with the PE heating rate. We therefore conclude that it is the $\mathrm{PAH}$ population that dominates the PE heating process.

The efficiency of the PE heating process is dependent on both environmental conditions, such as radiation field, and grain abundances. Our study has examined PE efficiency within the LMC without fully disentangling the extent of PE heating due to existence of grain populations favourable to PE heating and the extent due to environmental conditions favourable to PE heating. Disentangling the effects of both on the observed PE efficiency, however, is difficult as the regions where PAH populations are destroyed naturally have intense radiation fields which also suppress the extent of PE heating. To break this degeneracy we will undertake detailed SED modelling of the different regions in the LMC in an upcoming paper. Using the SED models we can independently solve for radiation field values and PAH abundances.

Acknowledgements. We would like to thank F. Boulanger, L. Verstraete and A. Jones for their helpful conversations. Meixner, Vijh, Sewilo and Leitherer have been funded by the NASA/Spitzer grant 1275598, and NASA NAG-12595.

\section{References}

Aguirre, J. E., Bezaire, J. J., Cheng, E. S., et al. 2003, ApJ, 596, 273 Aitken, D. K., \& Roche, P. F. 1985, MNRAS, 213, 777 Bakes, E. L. O., \& Tielens, A. G. G. M. 1994, ApJ, 427, 822 Bernard, J. P., Reach, W., \& Paradis, D. 2008, AJ, submitted
Cesarsky, D., Lequeux, J., Abergel, A., et al. 1996, A\&A, 315, L309 Dale, D. A., \& Helou, G. 2002, ApJ, 576, 159

Dalgarno, A., \& McCray, R. A. 1972, ARA\&A, 10, 375

de Jong, T. 1977, A\&A, 55, 137

Desert, F.-X., Boulanger, F., \& Puget, J. L. 1990, A\&A, 237, 215

Draine, B. T. 1978, ApJS, 36, 595

Draine, B. T., \& Li, A. 2001, ApJ, 551, 807

Draine, B. T., \& Li, A. 2007, ApJ, 657, 810

Dufour, R. J. 1984, in Structure and Evolution of the Magellanic Clouds, ed. S. van den Bergh, \& K. S. D. Boer, IAU Symp., 108, 353 Dwek, E., \& Smith, R. K. 1996, ApJ, 459, 686

Engelbracht, C. W., Rieke, G. H., Gordon, K. D., et al. 2008, ApJ, 678, 804 Fazio, G. G., Hora, J. L., Allen, L. E., et al. 2004, ApJS, 154, 10

Feast, M. 1999, in New Views of the Magellanic Clouds, ed. Y.-H. Chu,

N. Suntzeff, J. Hesser, \& D. Bohlender, IAU Symp., 190, 542

Ferriere, K. M., Zweibel, E. G., \& Shull, J. M. 1988, ApJ, 332, 984

Galliano, F., Dwek, E., \& Chanial, P. 2007, ArXiv e-prints, 708

Gaustad, J. E., McCullough, P. R., Rosing, W., \& Van Buren, D. 2001, PASP, 113,1326

Goldsmith, D. W., Habing, H. J., \& Field, G. B. 1969, ApJ, 158, 173

Habart, E., Verstraete, L., Boulanger, F., et al. 2001, A\&A, 373, 702

Heiles, C. 1994, ApJ, 436, 720

Helou, G. 1986, ApJ, 311, L33

Hollenbach, D. J., Takahashi, T., \& Tielens, A. G. G. M. 1991, ApJ, 377, 192

Hunter, D. A., Kaufman, M., Hollenbach, D. J., et al. 2001, ApJ, 553, 121

Israel, F. P., Maloney, P. R., Geis, N., et al. 1996, ApJ, 465, 738

Keller, S. C., \& Wood, P. R. 2006, ApJ, 642, 834

Kennicutt, Jr., R. C., \& Hodge, P. W. 1986, ApJ, 306, 130

Kim, S., \& Reach, W. T. 2002, ApJ, 571, 288

Leach, S. 1987, A\&A, 187, 195

Maciel, W. J., \& Pottasch, S. R. 1982, A\&A, 106, 1

Madden, S. C. 2000, New A Rev., 44, 249

Madden, S. C., Geis, N., Genzel, R., et al. 1993, ApJ, 407, 579

Madden, S. C., Poglitsch, A., Geis, N., Stacey, G. J., \& Townes, C. H. 1997, ApJ, 483, 200

Madden, S. C., Galliano, F., Jones, A. P., \& Sauvage, M. 2006, A\&A, 446, 877

Malhotra, S., Helou, G., Stacey, G., et al. 1997, ApJ, 491, L27

Malhotra, S., Kaufman, M. J., Hollenbach, D., et al. 2001, ApJ, 561, 766

Matsuhara, H., Nakagawa, T., Shibai, H., et al. 1989, ApJ, 339, L67

McKee, C. F., \& Ostriker, J. P. 1977, ApJ, 218, 148

Meixner, M., Haas, M. R., Tielens, A. G. G. M., Erickson, E. F., \& Werner, M. 1992, ApJ, 390, 499

Meixner, M., Gordon, K. D., Indebetouw, R., et al. 2006, AJ, 132, 2268

Mochizuki, K., Nakagawa, T., Doi, Y., et al. 1994, ApJ, 430, L37

Nordgren, T. E., Cordes, J. M., \& Terzian, Y. 1992, AJ, 104, 1465

Peck, A. B., Goss, W. M., Dickel, H. R., et al. 1997, ApJ, 486, 329

Petuchowski, S. J., \& Bennett, C. L. 1993, ApJ, 405, 591

Poglitsch, A., Krabbe, A., Madden, S. C., et al. 1995, ApJ, 454, 293

Povich, M. S., Stone, J. M., Churchwell, E., et al. 2007, ApJ, 660, 346

Rieke, G. H., Young, E. T., Engelbracht, C. W., et al. 2004, ApJS, 154, 25

Roche, P. F., \& Aitken, D. K. 1985, MNRAS, 213, 789

Röllig, M., Abel, N. P., Bell, T., et al. 2007, A\&A, 467, 187

Sakon, I., Onaka, T., Kaneda, H., et al. 2006, ApJ, 651, 174

Siebenmorgen, R., Krügel, E., \& Spoon, H. W. W. 2004, A\&A, 414, 123

Spitzer, L. J. 1948, ApJ, 107, 6

Stacey, G. J., Geis, N., Genzel, R., et al. 1991, ApJ, 373, 423

Steiman-Cameron, T. Y., Haas, M. R., Tielens, A. G. G. M., \& Burton, M. G. 1997, ApJ, 478, 261

Stutzki, J., Stacey, G. J., Genzel, R., et al. 1988, ApJ, 332, 379

Tielens, A. G. G. M. 2005, The Physics and Chemistry of the Interstellar Medium ed. A. G. G. M. Tielens (University Press: Cambridge, UK: Cambridge)

Tielens, A. G. G. M., \& Hollenbach, D. 1985a, ApJ, 291, 747

Tielens, A. G. G. M., \& Hollenbach, D. 1985b, ApJ, 291, 722

Valls-Gabaud, D. 1998, PASA, 15, 111

Vermeij, R., Damour, F., van der Hulst, J. M., \& Baluteau, J. P. 2002a, A\&A, 390, 649

Vermeij, R., Peeters, E., Tielens, A. G. G. M., \& van der Hulst, J. M. 2002b, A\&A, 382, 1042

Verstraete, L., Pech, C., Moutou, C., et al. 2001, A\&A, 372, 981

Voit, G. M. 1992, MNRAS, 258, 841

Watson, W. D. 1972, ApJ, 176, 103

Weingartner, J. C., \& Draine, B. T. 2001, ApJS, 134, 263

Westerlund, B. E. 1997, The Observatory, 117, 317

Wolfire, M. G., Tielens, A. G. G. M., \& Hollenbach, D. 1990, ApJ, 358, 116

Wright, E. L., Mather, J. C., Bennett, C. L., et al. 1991, ApJ, 381, 200

Wu, Y., Charmandaris, V., Hao, L., et al. 2006, ApJ, 639, 157

Young Owl, R. C., Meixner, M. M., Fong, D., et al. 2002, ApJ, 578, 885 